\title{
Synthesis of Butenolides via a Horner-Wadsworth-Emmons cascading dimerization reaction
}

\section{Jack Everson and Milton J Kiefel*}

Institute for Glycomics, Griffith University Gold Coast Campus, Southport, Queensland, 4222, Australia

Email: m.kiefel@griffith.edu.au

Table of Contents Graphic

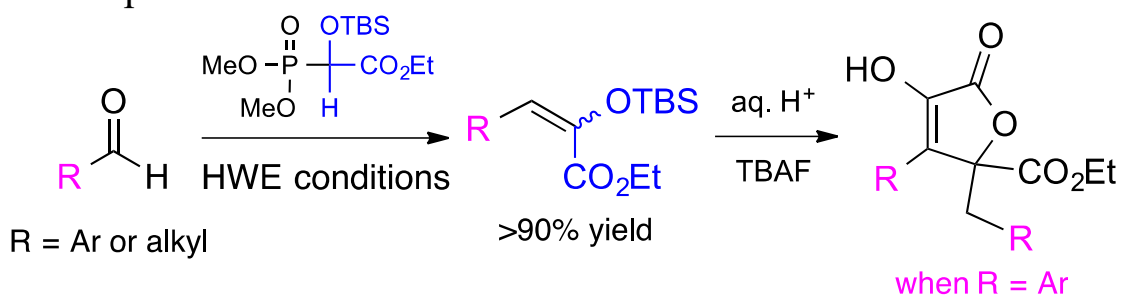

\begin{abstract}
The efficient synthesis of a range of structurally related butenolides has been observed whilst we were exploring the substrate-scope of a Horner-Wadsworth-Emmons (HWE) reaction. Whilst aliphatic aldehydes gave the expected HWE product, aromatic aldehydes furnished butenolides, resulting from the dimerization of HWE product during desilylation of the initially formed HWE adduct. In addition to isolating butenolides in high yield, we have also determined precisely when dimerization occurs.
\end{abstract}

\section{INTRODUCTION}

The Horner-Wadsworth-Emmons (HWE) reaction has long been one of the favoured methods for the olefination of carbonyl compounds, due to the robustness, high stereoselectivity, and the varied substrate scope of the reaction. The HWE reaction forms the cornerstone of many natural product syntheses (see for example, reviews by Kobayashi et al., ${ }^{1}$ Burtoloso et al., ${ }^{2}$ Al Jasem et $a l .,{ }^{3}$ and Bisceglia \& Orelli, ${ }^{4}$ as well some recent examples ${ }^{5,6}$ ), and is particularly valuable in carbon chain elongation, closing macrocyclic rings, ${ }^{7}$ and the coupling of synthetic segments. ${ }^{8}$ The HWE reaction is also valuable in generating vinyl sulfones, ${ }^{9}$ and chiral sulfoxides. ${ }^{10}$

Our interest in the HWE reaction is based upon the ability to use this important carbon-carbon bond forming reaction to generate chain elongated carbohydrate analogues. We have previously described our efforts towards developing new synthetic approaches towards the nine-carbon acidic 
sugars known as nonulosonic acids, and have successfully described the synthesis of pseudaminic acid and legionaminic acid analogues. ${ }^{11-13}$ Nonulosonic acids are found as key components of glycans in several pathogenic Gram-negative bacteria, notably Campylobacter,${ }^{14}$ Pseudomonas, ${ }^{15}$ Acinetobacter,${ }^{16}$ Helicobacter,${ }^{17}$ and Legionella.${ }^{18,19}$ Significantly, nonulosonic acids are known to be directly associated with the virulence of these pathogenic bacteria. ${ }^{19-22}$

The synthesis of complex higher order sugars requires approaches either starting from existing higher order sugars, ${ }^{23}$ or using approaches based on carbon-carbon bond forming reactions. ${ }^{24}$ With respect to the latter strategy, in 2015 Feng et al. described the efficient large scale synthesis of KDO, an 8-carbon acidic sugar found in all Gram-negative bacteria, from D-mannose. ${ }^{25}$ Key to the success of this synthesis was the use of a HWE reaction between the protected mannose derivative $\mathbf{1}$ and the phosphonate $\mathbf{2}$, to give the HWE adduct $\mathbf{3}$ which furnished the KDO ethyl ester derivative 4 after desilyation (Scheme 1). Previously we have reported the synthesis of a series of structurally modified KDO derivatives, starting from ribose and using an aldol condensation. ${ }^{26}$ The publication summarized in Scheme 1 was therefore particularly interesting to us, and we wondered if it was possible to adapt the chemistry described by Feng et al. to include structurally modified mannose derivatives, or indeed other types of sugars.

Scheme 1. Synthesis of the KDO ethyl ester derivative 4 using a HWE condensation. ${ }^{25}$

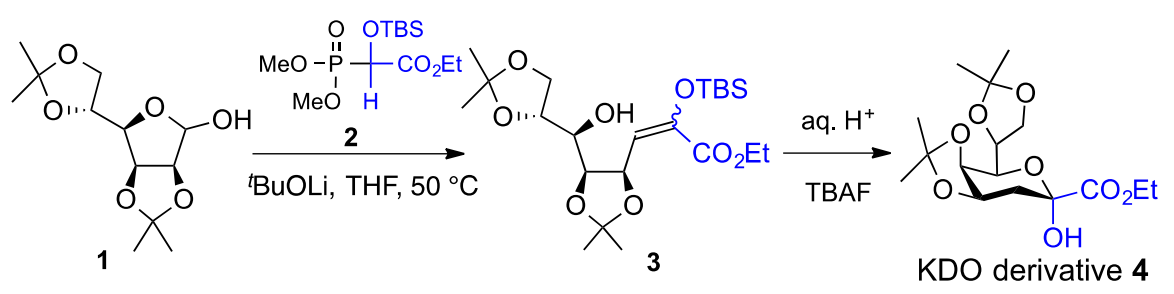

\section{RESULTS AND DISCUSSION}

Our initial studies into exploring the potential of using the HWE chemistry described by Feng et $a l .{ }^{25}$ for the synthesis of higher order sugars started with some mannose derivatives, notably those modified at C-6. The preparation of these C-6 modified mannose derivatives is relatively straightforward, commencing with the bis-isopropylidenated mannose derivative 1, selective 
removal of the 5,6-isopropylidene group (90\% aq. $\mathrm{AcOH})$, and subsequent selective modification of the primary C-6 hydroxyl. In this way we prepared the modified mannose derivatives 5a-c shown in Table 1, and then exposed each of them to the phosphate ester 2 under the HWE conditions described by Feng et al. ${ }^{25}$ We were delighted to note that the C-8 azido KDO derivative $\mathbf{6 b}(54 \%)$ could be obtained in good yield from the corresponding 6-azido mannose derivative $\mathbf{5 b}$ (Table 1), showing that an unprotected $\mathrm{C} 5-\mathrm{OH}$ group was not a problem. Unfortunately, the 5,6diol derivative (Table 1, entry 3) did not furnish any of the corresponding KDO product, which is perhaps due to the increased acidity of the primary hydroxyl group.

Table 1. Synthesis of KDO derivatives 6 via a HWE condensation with phosphate ester 2.

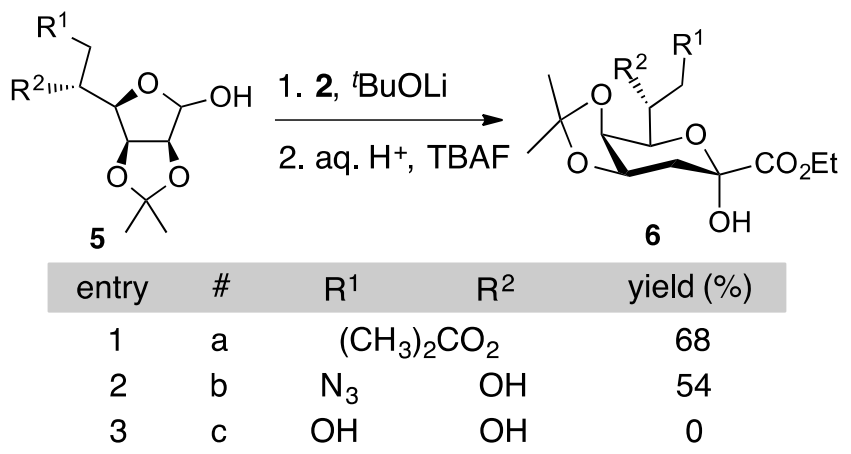

Interestingly, alternative carbohydrates such as 2,3,4-tri- $O$-benzylglucose and 2,3,5,6-tetra- $O$ acetylgalactofuranose failed to furnish any of the corresponding 8-carbon sugar product under the same HWE conditions as those shown in Table 1. The failure of these alternative carbohydrates to be substrates led us to consider if this particular HWE reaction was only effective on mannosebased substrates, since clearly the nature of the sugar substrate will reflect the preference for it to exist in the acyclic form needed to react with the phosphate ester 2.

Given our ongoing interest in developing novel and efficient syntheses of higher order carbohydrates, ${ }^{11-13}$ we thought it would be valuable to explore the scope of this HWE reaction on simple aldehydes. Accordingly, a series of commercially available aromatic and aliphatic aldehydes were reacted with the phosphonate ester $\mathbf{2}$ under the same conditions described by Feng et al. Each of the HWE adducts 7 obtained were purified using column chromatography and 
characterized spectroscopically. As can be seen in Table 2, all the commercially available aldehydes we used furnished the HWE adducts 7 in essentially quantitative yield after chromatographic purification. We observed no real differences in rates of reaction or in efficiency of the HWE condensation, irrespective of electron rich, electron deficient, aromatic, or aliphatic aldehydes.

Table 2. Results from the HWE condensation between phosphate ester 2 and aldehydes.

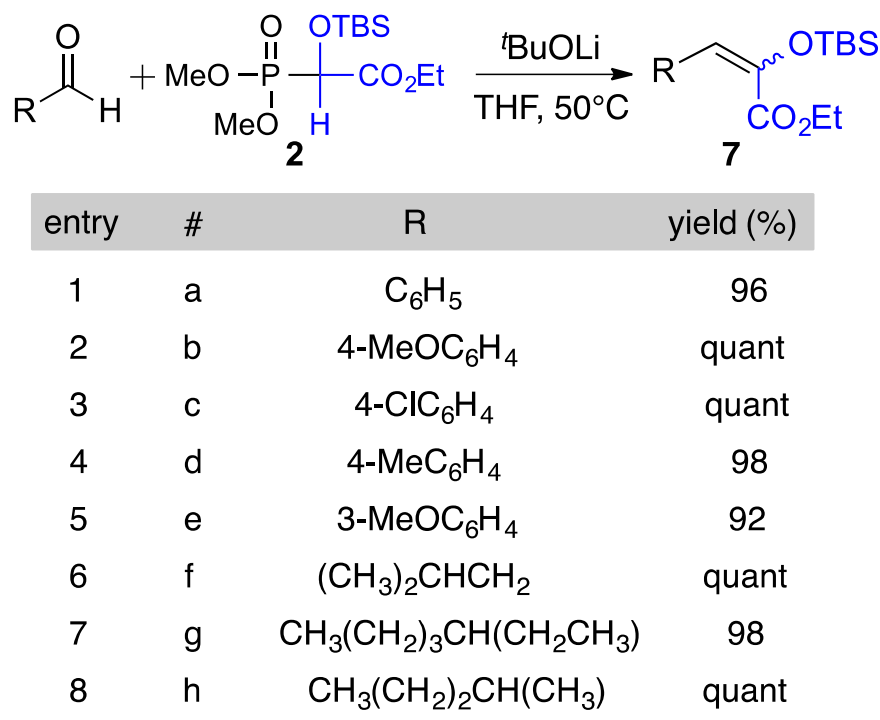

Unexpectedly, desilylation of the HWE adduct $7 \mathbf{b}\left(\mathrm{R}=4-\mathrm{MeOC}_{6} \mathrm{H}_{4}\right)$ under the conditions described by Feng et al. $^{25}$ (TBAF and aqueous AcOH) and subsequent neutralization and purification did not give any of the expected $\alpha$-keto-ester product 8. Careful analysis of the spectroscopic data of the single product obtained, most importantly the ${ }^{1} \mathrm{H}$ NMR and ${ }^{13} \mathrm{C}$ NMR spectra, clearly showed that there were two different 4-MeO-phenyl rings in the product. Key pieces of spectroscopic evidence that led to the final structure $9 \mathbf{b}$ being proposed (Scheme 2) included the observation of a single ethyl ester group ( $\delta 4.17 \mathrm{ppm}, \mathrm{q}, 2 \mathrm{H}$, and $\delta 1.14 \mathrm{ppm}, \mathrm{t}, 3 \mathrm{H})$ in the ${ }^{1} \mathrm{H}$ NMR, but the presence of two ester-type carbons ( $\delta 169.3 \mathrm{ppm}$ and $\left.169.1 \mathrm{ppm}\right)$ in the ${ }^{13} \mathrm{C}$ spectrum. Based on the ${ }^{1} \mathrm{H}$ and ${ }^{13} \mathrm{C}$ data, and a $m / z$ of $421(\mathrm{M}+\mathrm{Na})$ in the ESI mass spectrum of $9 \mathbf{b}$, it was clear that we had formed a dimer of the desilylated HWE adduct with concomitant loss of ethanol. Careful inspection of the long-range ${ }^{1} \mathrm{H}-{ }^{13} \mathrm{C} \mathrm{HMBC}$ spectrum provided the key 
evidence to arrive at structure $\mathbf{9 b}$, notably a correlation from both the ethyl ester carbonyl carbon ( $\delta 169.1 \mathrm{ppm})$ and the butenolide ring carbon $(\delta 86.2 \mathrm{ppm})$ into the benzylic $\mathrm{CH}_{2}$ resonance (an $\mathrm{AB}$ spin system centred at $\delta 3.55 \mathrm{ppm})$.

Scheme 2. Unexpected dimerization of HWE adducts upon desilylation.

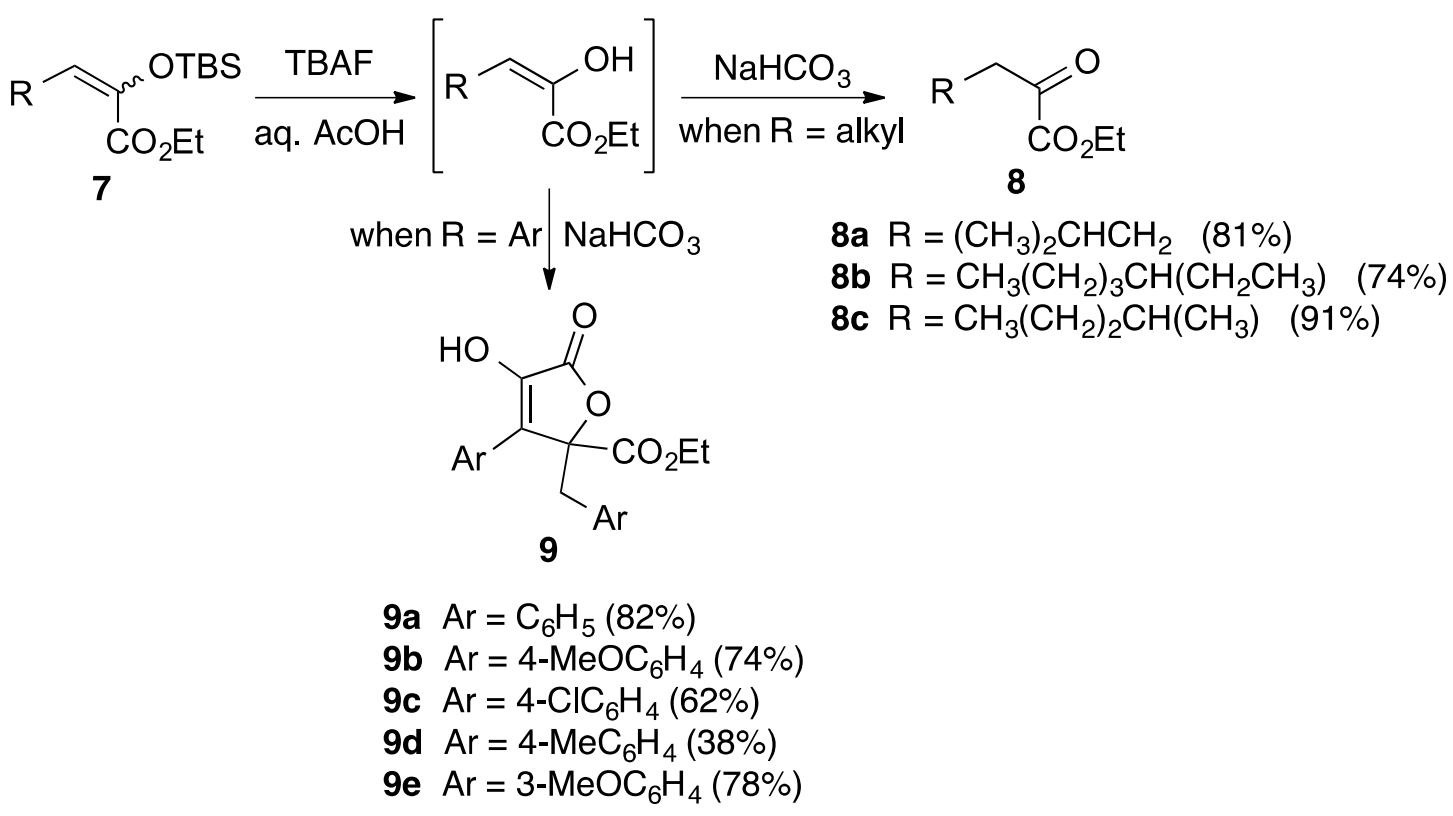

The butenolide $9 \mathbf{b}\left(\mathrm{Ar}=4-\mathrm{MeOC}_{6} \mathrm{H}_{4}\right)$ was obtained in high yield $(74 \%)$ from the corresponding HWE adduct $\mathbf{7 b}$ under the desilylation conditions described. Interestingly, all the aromatic-based HWE adducts 7a-e gave the corresponding dimeric butenolide structures $\mathbf{9}$ as the only identifiable product in modest to high yield (Scheme 2) after desilylation, whilst the aliphatic-based HWE adducts 7f-h only furnished the expected monomeric $\alpha$-keto-ester products 8a-c (Scheme 2).

We believe the unexpected formation of the butenolides 9 during the desilylation of the HWE adducts 7 that are aromatic-based, but not aliphatic, is due to the propensity of the desilylated aromatic-based compounds to exist in both their keto and enol forms. Careful analysis (by TLC) of the reaction mixture during desilylation clearly showed that dimerization only occurs upon neutralization (with $\mathrm{NaHCO}_{3}$ ) of the reaction mixture. This observation, together with the lack of 
dimerisation observed during the desilylation of the aliphatic-based HWE adducts 7, we propose that the formation of the butenolides 9 occurs by the mechanism depicted in Figure 1. As can be seen, desilylation of the aromatic-based substrates leads to a mixture of the keto- and enol-forms of the HWE adducts. Upon neutralization with $\mathrm{NaHCO}_{3}$ (to $\mathrm{pH} \approx 8$ ), the enol form attacks the keto form to give the dimeric intermediate 10, which then undergoes an internal attack by the newly generated oxygen anion, resulting in loss of ethanol to give the butenolides 9 after ketoenol tautomerism. Whilst the dimerization of $\alpha$-keto esters under base catalysis is known (see Scheme 3 and associated discussion below), it is interesting to note that we observe a difference in behaviour between aryl and alkyl HWE adducts like 7, suggesting that perhaps this is not just a simple case of base catalysing the dimerization.

Figure 1. Mechanism for the formation of the butenolides 9 from the HWE adducts 7.

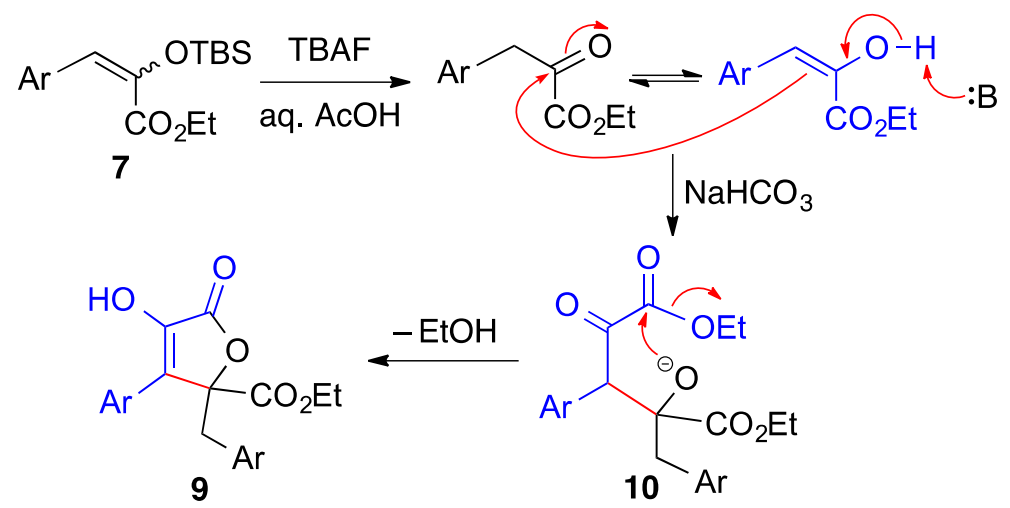

Butenolides such as $\mathbf{9}$ are an interesting class of natural product often isolated from fungal sources. ${ }^{27,28}$ Of particular interest to us is the fact that several butenolides like 9 have been shown to have biological activity. The butenolide 11 (Figure 2), isolated from Aspergillus terreus, ${ }^{29}$ is an inhibitor of CDK2 and CDK1 kinases, showing antiproliferative activity against pancreatic and prostate cancer cell lines. ${ }^{30,31}$ A recent study isolated 12 different butenolides from A. terreus, ${ }^{32}$ and showed that in addition to $\mathbf{1 1}$, the "symmetrical" butenolide $\mathbf{1 2}$ (i.e. a homodimer similar to 9) also showed promising activity against pancreatic cancer. Butenolides like 13, isolated from an endophytic Aspergillus from Tripterygium wilfordii, have shown anti-inflammatory activity. ${ }^{28}$ 
Figure 2. Naturally occurring butenolides with biological activity.

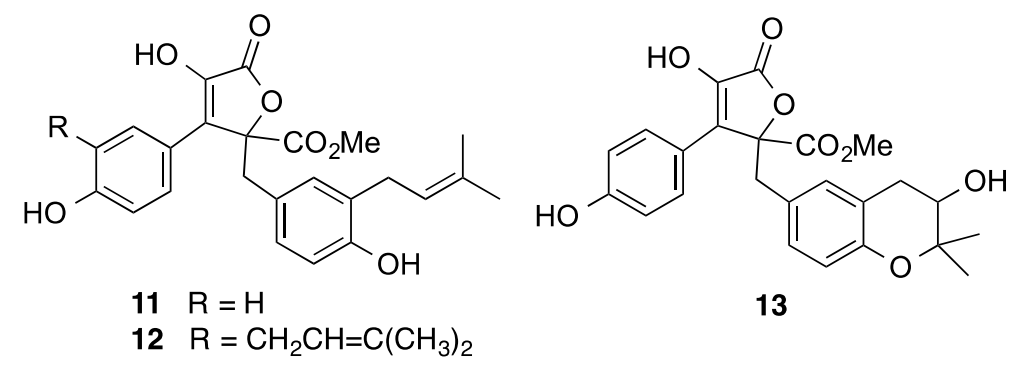

It is interesting to note that butenolides with the most potent anticancer activity tend to be asymmetrical with respect to the aromatic rings. Indeed, in the work by Braña et al., ${ }^{31}$ the synthetic butenolide analogue of $\mathbf{1 1}$ that lacked the 3-methylbut-2-enyl chain had no activity against a range of human cancer cell lines. Since it appears that asymmetrical butenolides have more potent biological activity, we speculated if we could obtain an unsymmetrical butenolide by exposing two different HWE adducts to the desilylation conditions. Accordingly, exposure of an equimolar mixture of the aryl HWE adduct $\mathbf{7 b}$ and the alkyl HWE adduct $\mathbf{7 h}$ to TBAF and aqueous AcOH, followed by neutralization $\left(\mathrm{NaHCO}_{3}\right.$, to $\left.\mathrm{pH} \approx 8\right)$ resulted in the isolation of, perhaps not surprisingly, a complex mixture of products. Careful chromatographic purification showed that we had indeed obtained the non-symmetrical butenolide $\mathbf{1 4}(\mathrm{m} / \mathrm{z}=385[\mathrm{M}+\mathrm{Na}])$ as a mixture of diastereomers, along with the symmetrical butenolide $\mathbf{9 b}$ and the $\alpha$-keto-ester product $\mathbf{8 c}$.

Scheme 3. Formation of the unsymmetrical butenolide 14.

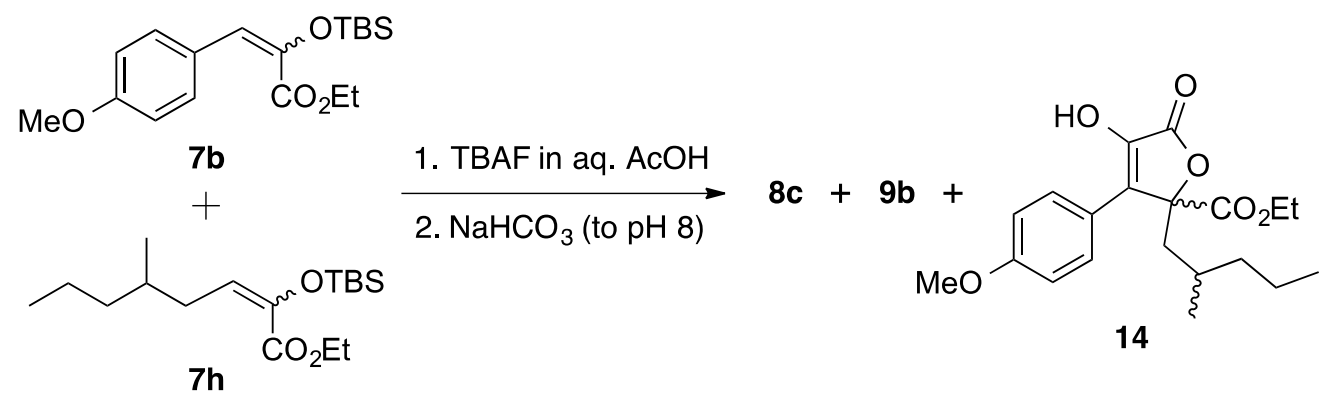

Obtaining only the single non-symmetrical butenolide $\mathbf{1 4}$ from the two HWE adducts $\mathbf{7 b}$ and 7h as depicted in Scheme 3 was not unexpected, since we have already shown (vide infra) that the alkyl HWE adducts do not form butenolides. Exploring this concept of preparing non-symmetrical 
butenolides further, we exposed an equimolar mixture of the two aromatic HWE adducts $7 \mathbf{a}$ and 7b to the desilylation conditions (Scheme 4). After neutralisation we obtained, as expected, a complex mixture of products in essentially statistical proportions, whereby each HWE adduct can dimerize with itself (giving 9a and 9b), and can also dimerise with the other reactant in solution, giving $\mathbf{1 5}$ and $\mathbf{1 6}$ as an inseparable mixture. We considered that it may be possible to control the dimerization between two different aryl HWE adducts by desilylation of one of the components to give the $\alpha$-keto-ester followed by exposure to a different aryl HWE adduct under the desilylation/dimerization conditions. However, desilylation of the aryl HWE adduct $\mathbf{7 b}$ without neutralisation gave the expected product 17 which exists, as reported by others, ${ }^{33}$ exclusively in its enol form. Nonetheless, exposure of $\mathbf{1 7}$ to the HWE adduct $\mathbf{7 a}$ under the standard desilylation/dimerization conditions gave the same mixture of products (Scheme 4), in comparable ratio as to that obtained when $7 \mathbf{a}$ and $\mathbf{7 b}$ were reacted together.

\section{Scheme 4. Formation of the unsymmetrical butenolides $15 \& 16$.}

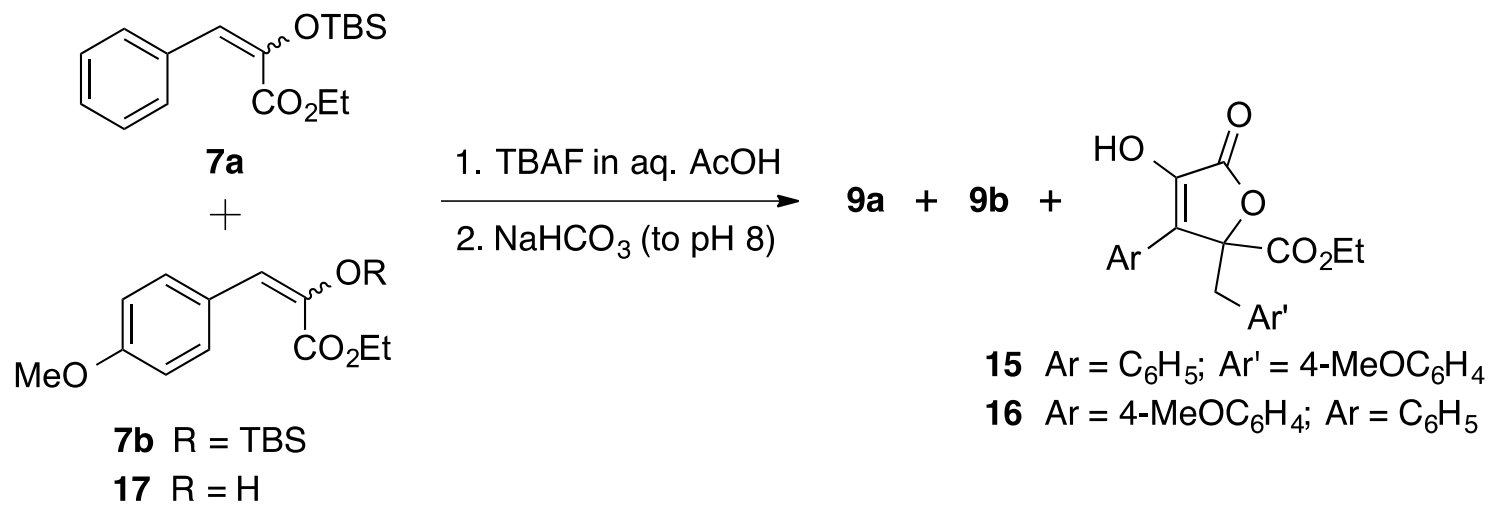

Given the interesting biological activity of these butenolides, especially the activity against pancreatic cancer, it is not surprising that several approaches towards the synthesis of analogues of these natural products is of interest. The synthesis of butenolides has been accomplished using metal-catalysed cyclisation approaches,${ }^{34}$ radical cyclisations, ${ }^{35}$ acid-catalysed lactonization, ${ }^{36}$ hydrolysis and saponification of quinones, ${ }^{37}$ and in enantiomerically pure form. ${ }^{38,39}$ For symmetrical butenolides like 9, the most common synthetic approach tends to mimic the 
postulated biosynthetic pathway, ${ }^{27,40}$ wherein simple aryl pyruvic methyl esters are dimerized in the presence of base. A representative example is summarized in Scheme 5. ${ }^{31}$ In that work, the aryl pyruvic methyl esters $\mathbf{1 8}$ themselves were obtained in either 3 or 4 steps from commercially available aldehydes or nitriles, respectively, and then treated with either $\mathrm{K}_{2} \mathrm{CO}_{3}$ in acetone or DBU in DMF to furnish the corresponding symmetric butenolides in yields typically $50-80 \%{ }^{31}$

\section{Scheme 5. Synthesis of butenolides via dimerization of $\alpha$-keto esters. ${ }^{31}$}
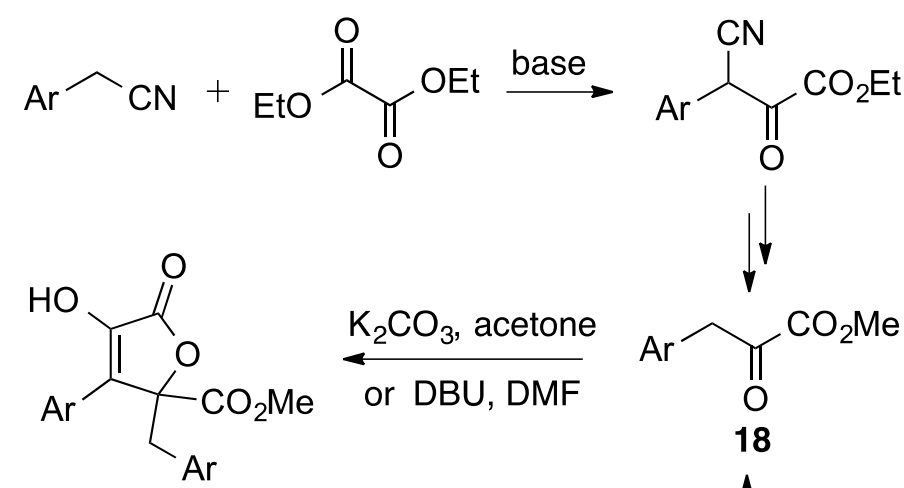

18<smiles>[3H][CH]</smiles><smiles>CC(=O)NCC(=O)ONC(=O)O[Te]C=C1N=C(C)OC1=O</smiles>

In comparison to the current literature processes, our synthesis of butenolides 9 therefore represents a very simple and high yielding approach towards these important natural products, since the phosphonate ester $\mathbf{2}$ is easy to prepare, and the aromatic aldehydes are commercially available. In order to further explore the scope of this unexpected butenolide formation, we exposed aromatic aldehydes (benzaldehyde, 4-methoxy- and 4-chlorobenzaldehyde) to the phosphate ester 2 under the standard HWE conditions, and then without purification treated the crude HWE adducts 7 under desilylation conditions. In each case, the butenolides 9 were obtained in high yield $\left(84 \%\right.$, for $\mathrm{Ar}=\mathrm{C}_{6} \mathrm{H}_{5}, 75 \%$ for $\mathrm{Ar}=4-\mathrm{MeOC}_{6} \mathrm{H}_{4}, \& 67 \%$ for $\left.\mathrm{Ar}=4-\mathrm{ClC}_{6} \mathrm{H}_{4}\right)$.

\section{CONCLUSIONS}

In summary, our synthesis of aromatic butenolides $\mathbf{9}$ directly from the corresponding aromatic aldehyde in 2 steps and without the need for purification of the intermediate HWE adduct 7 
represents a highly efficient and simple method for synthesizing this important class of natural product. Further studies exploring different aldehydes and the possibility of making unsymmetrical butenolides are in progress in our laboratory.

\section{EXPERIMENTAL SECTION}

General Information: Unless stated otherwise, all commercially available reagents were utilized without further purification. All "anhydrous" solvents were either purchased directly from Sigma, or were dried following a procedure described in Armarego \& Chai. ${ }^{41}$ 'Flash' chromatography using silica gel 60 was performed routinely in order to purify all products. All heating was performed using a magnetic stirrer hotplate and an appropriate sized heating block. Low resolution mass spectral (LRMS) analysis was performed using a Bruker esquire 3000 electrospray ionisation mass spectrometer and high resolution mass spectrometry (HRMS) was performed using a Bruker MaXis II quadrupole time of flight electrospray ionisation mass spectrometer. IR spectral analysis was performed using a Bruker Alpha Fourier Transform Infra-Red spectrometer. ${ }^{1} \mathrm{H}$ and ${ }^{13} \mathrm{C}$ NMR spectra were obtained using a Bruker $400 \mathrm{MHz}$ spectrometer at 400 and $100 \mathrm{MHz}$, respectively. Signals are reported in terms of their chemical shift ( $\delta$ in ppm) relative to the deuterated solvent used to obtain that spectrum. All ${ }^{13} \mathrm{C}$ NMR spectra were recorded using a DEPTQ-135 pulse sequence resulting in quaternary and methylene carbon resonances phased negative and methine and methyl carbons phased positive.

\section{Ethyl ester $\alpha$-(dimethylphosphinyl) glycolate t-butyldimethylsilyl ether (2)}

Dimethylphosphite $(6.8 \mathrm{~g}, 5.7 \mathrm{~mL}, 62.3 \mathrm{mmol})$ was dissolved in dry toluene $(70 \mathrm{~mL})$ under argon. The reaction mixture was cooled to $0^{\circ} \mathrm{C}$ (ice bath) and $\mathrm{Et}_{3} \mathrm{~N}(14.5 \mathrm{~g}, 20 \mathrm{~mL}, 143.7 \mathrm{mmol})$ was added. After $15 \mathrm{mins} 50 \%$ ethylglyoxalate hydrate in toluene ( $6 \mathrm{~g}, 12 \mathrm{~mL}, 47.9 \mathrm{mmol})$ was added and the reaction mixture was warmed to room temperature. The reaction mixture was then stirred for 3 hours before being concentrated in vacuo. The crude residue was then dissolved in dry DMF $(60 \mathrm{~mL})$ under argon. Imidazole $(6.5 \mathrm{~g}, 95.8 \mathrm{mmol})$ was then added followed by TBDMSCl $(11.6$ $\mathrm{g}, 76.6 \mathrm{mmol})$. The reaction mixture was stirred overnight at room temperature. The reaction mixture was then diluted with EtOAc $(100 \mathrm{~mL})$, washed with sat. aq. $\mathrm{NaHCO}_{3}$, dried $\left(\mathrm{Na}_{2} \mathrm{SO}_{4}\right)$ and filtered. The organic phase then concentrated under vacuum. The residue was purified via column chromatography (1:2, EtOAc:hexane, v/v, Rf: 0.28$)$ to give the known ${ }^{25}$ phosphonate $(12.5$ g, $38.3 \mathrm{mmol}, 80 \%$ yield) as a clear, viscous liquid. 
${ }^{1} \mathrm{H} \mathrm{NMR}\left(\mathrm{CDCl}_{3}, 400 \mathrm{MHz}\right): \delta(\mathrm{ppm}) 4.60(1 \mathrm{H}, \mathrm{d}, J=17.6 \mathrm{~Hz}), 4.32-4.20$ (2H, m), 3.84 (3H, d, $J$ $=6.7 \mathrm{~Hz}), 3.81(3 \mathrm{H}, \mathrm{d}, J=6.7 \mathrm{~Hz}), 1.29(3 \mathrm{H}, \mathrm{t}, J=7.1 \mathrm{~Hz}), 0.91(9 \mathrm{H}, \mathrm{s}), 0.11(3 \mathrm{H}, \mathrm{s}), 0.10(3 \mathrm{H}$, s). ${ }^{13} \mathrm{C}$ NMR $\left\{\right.$ DEPTQ-135\} $\left(\mathrm{CDCl}_{3}, 100 \mathrm{MHz}\right) \delta(\mathrm{ppm}) 168.5(\mathrm{~d}, J=3.1 \mathrm{~Hz}, \mathrm{P}$ split), 71.4, 69.8 (d, $J=162.0 \mathrm{~Hz}, \mathrm{P}$ split), 61.8, 54.1 (t, $J=6.7 \mathrm{~Hz}, \mathrm{P}$ split), 25.5, 18.4, 14.1, -5.2, -5.4. The ${ }^{1} \mathrm{H}$ and ${ }^{13} \mathrm{C}$ NMR data is consistent with published NMR data. ${ }^{25}$ IR (neat): 2955, 2930, 2895, 2855, 1750 $\mathrm{cm}^{-1}$. LRMS (ESI) $m / z: 349[\mathrm{M}+\mathrm{Na}]^{+}$.

\section{2,3:5,6-di-O-isopropylidene- $\alpha, \beta$-D-mannose $(1)^{25}$}

D-Mannose (5.0 g, $27.8 \mathrm{mmol})$ was placed under argon and dissolved in dry DMF (50 mL). To this was added $\mathrm{TsOH} \cdot \mathrm{H}_{2} \mathrm{O}(0.32 \mathrm{~g}, 1.7 \mathrm{mmol})$ followed by 2,2-dimethoxypropane $(8.5 \mathrm{~g}, 10.0 \mathrm{~mL}$, $83.3 \mathrm{mmol})$. The reaction mixture was stirred at room temperature overnight. The reaction mixture was then diluted with EtOAc $(\sim 100 \mathrm{~mL})$ and was washed with saturated $\mathrm{NaHCO}_{3}(\sim 50 \mathrm{~mL})$. The aqueous phase was then extracted with EtOAc $(3 \times 50 \mathrm{~mL})$ and all organic phases were combined. The organic phases were dried $\left(\mathrm{Na}_{2} \mathrm{SO}_{4}\right)$, filtered and then concentrated under reduced pressure. The crude residue was recrystallised (EtOAc/hexane) to give the known ${ }^{25}$ title compound 1 (5.5 g, $21.1 \mathrm{mmol}, 76 \%)$ as a white crystalline powder.

${ }^{1} \mathrm{H}$ NMR $\left(\mathrm{CDCl}_{3}, 400 \mathrm{MHz}\right): \delta(\mathrm{ppm}) 5.38(1 \mathrm{H}, \mathrm{d}, J=2.3 \mathrm{~Hz}, \mathrm{H} 1), 4.81(1 \mathrm{H}, \mathrm{dd}, J=5.6 \mathrm{~Hz}, 3.6$ $\mathrm{Hz}, \mathrm{H} 3), 4.62$ (1H, d, $J=5.6 \mathrm{~Hz}, \mathrm{H} 2), 4.43-4.38$ (1H, m, H5), 4.19 (1H, dd, $J=7.2,3.6 \mathrm{~Hz}, \mathrm{H} 4)$, 4.09 (1H, dd, $J=8.7,6.3 \mathrm{~Hz}, \mathrm{H6}), 4.04$ (1H, dd, $J=8.6,4.8 \mathrm{~Hz}, \mathrm{H6}$ '), 1.47, 1.46, 1.38, 1.33 (4 x $\left.3 \mathrm{H}, 4 \mathrm{x} \mathrm{s}, 4 \mathrm{x}\left(\mathrm{CH}_{3}\right)_{2} \mathrm{CO}\right) .{ }^{13} \mathrm{C} \mathrm{NMR}\{\mathrm{DEPTQ}-135\}\left(\mathrm{CDCl}_{3}, 100 \mathrm{MHz}\right) \delta(\mathrm{ppm}) 112.8,109.2(2 \mathrm{x}$ $\left(\mathrm{CH}_{3}\right)_{2} \mathrm{CO}$ ), 101.4 (C1), 85.6, 80.5, 79.8, 73.4 (all CH, C2, C3, C4, C5), 66.7 (C6), 27.0, 26.0, 25.3, 24.6 (4 x $\left.\left(\mathrm{CH}_{3}\right)_{2} \mathrm{CO}\right)$, Note that trace amounts of the anomer can be observed in both ${ }^{1} \mathrm{H}$ and ${ }^{13} \mathrm{C}$ NMR spectra. Our ${ }^{1} \mathrm{H}$ and ${ }^{13} \mathrm{C}$ NMR data is consistent with published NMR data. ${ }^{25} \mathrm{IR}$ (neat): 3431, 2986, 2947, $2898 \mathrm{~cm}^{-1}$. LRMS (ESI) $\mathrm{m} / \mathrm{z}: 283[\mathrm{M}+\mathrm{Na}]^{+}$.

\section{2,3-O-Isopropylidene- $\alpha, \beta-D-m a n n o s e ~(5 c)^{42}$}

2,3:5,6-Di-O-isopropylidene- $\alpha, \beta$-D-mannose (1.4 g, $5.38 \mathrm{mmol})$ was dissolved in $30 \mathrm{~mL}$ of $90 \%$ $\mathrm{AcOH} / \mathrm{H}_{2} \mathrm{O}$. The reaction mixture was stirred for 5.5 hours and was then diluted with an excess of toluene. The reaction mixture was then placed under reduced pressure to remove solvent. The residue was diluted with toluene and then concentrated in vacuo a number of times to remove any remaining AcOH. The resulting residue was purified via column chromatography (9:1, EtOAc:MeOH, v/v, Rf: 0.15) to give the known ${ }^{42}$ 5,6-diol 5c (0.763 g, $\left.3.46 \mathrm{mmol}, 65 \%\right)$ as a clear, viscous liquid.

${ }^{1} \mathrm{H}$ NMR $\left(\mathrm{CDCl}_{3}, 400 \mathrm{MHz}\right): \delta(\mathrm{ppm}) 5.37$ (1H, brs, H1), $4.88(1 \mathrm{H}, \mathrm{dd}, J=5.6,4.0 \mathrm{~Hz}, \mathrm{H} 3), 4.61$ $(1 \mathrm{H}, \mathrm{d}, J=5.6 \mathrm{~Hz}, \mathrm{H} 2), 4.17$ (1H, dd, $J=8.6,3.7 \mathrm{~Hz}, \mathrm{H} 4), 4.00-3.95$ (1H, m, H5), 3.83 (1H, dd, 
$J=11.7,3.0 \mathrm{~Hz}, \mathrm{H} 6), 3.75\left(1 \mathrm{H}, \mathrm{dd}, J=11.7,4.6 \mathrm{~Hz}, \mathrm{H} 6\right.$ '), 1.46, $1.33\left(2\right.$ x 3H, 2 x s, 2 x $\left.\left(\mathrm{CH}_{3}\right)_{2} \mathrm{CO}\right)$. ${ }^{13} \mathrm{C} \mathrm{NMR}\left(\mathrm{CDCl}_{3}, 100 \mathrm{MHz}\right) \delta(\mathrm{ppm}) 112.8\left(\left(\mathrm{CH}_{3}\right)_{2} \mathrm{CO}\right), 101.1(\mathrm{C} 1), 85.5,80.1,79.1,70.1$ (all $\mathrm{CH}, \mathrm{C} 2, \mathrm{C} 3, \mathrm{C} 4, \mathrm{C} 5), 64.1(\mathrm{C} 6), 26.1,24.8\left(2 \times\left(\underline{\mathrm{CH}}_{3}\right)_{2} \mathrm{CO}\right)$, Note that trace amounts of the anomer can be observed in both ${ }^{1} \mathrm{H}$ and ${ }^{13} \mathrm{C}$ NMR spectra. Our ${ }^{1} \mathrm{H}$ and ${ }^{13} \mathrm{C}$ NMR data is consistent with published NMR data. ${ }^{40}$ LRMS (ESI) $m / z: 243[\mathrm{M}+\mathrm{Na}]^{+}$.

\section{2,3-O-Isopropylidene-6- $O$-( $(p$-toluenesulfonyl)- $\alpha, \beta$-D-mannose}

2,3-O-Isopropylidene- $\alpha, \beta$-D-mannose (5c) $(2.0 \mathrm{~g}, 9.08 \mathrm{mmol})$ was placed under argon and was dissolved in pyridine $(40 \mathrm{~mL})$. The reaction mixture was cooled to $0^{\circ} \mathrm{C}$ with an ice bath and tosyl chloride (2.6 g, $13.6 \mathrm{mmol})$ was added. The reaction mixture was warmed to room temperature and stirred overnight, before being concentrated in vacuo. The residue was dissolved in EtOAc $(100 \mathrm{~mL})$ and washed with dilute aqueous $\mathrm{HCl}(1 \mathrm{M}, 40 \mathrm{~mL})$, water $(40 \mathrm{~mL})$, dried $\left(\mathrm{Na}_{2} \mathrm{SO}_{4}\right)$, and concentrated under reduced pressure. The resulting residue was purified via column chromatography (3:2, hexane:EtOAc, v/v, Rf: 0.30$)$ to give the 6 - $O$-tosyl mannose derivative (1.86 $\mathrm{g}, 4.97 \mathrm{mmol}, 55 \%)$ as a white solid.

${ }^{1} \mathrm{H} \mathrm{NMR}\left(\mathrm{CDCl}_{3}, 400 \mathrm{MHz}\right): \delta(\mathrm{ppm}) 7.83(2 \mathrm{H}, \mathrm{d}, J=8.3 \mathrm{~Hz}, \mathrm{ArH}), 7.35$ (2H, d, $\left.J=8.3 \mathrm{~Hz}, \mathrm{ArH}\right)$, $5.34(1 \mathrm{H}, \mathrm{d}, J=2.2 \mathrm{~Hz}, \mathrm{H} 1), 4.85(1 \mathrm{H}, \mathrm{dd}, J=5.3,3.6 \mathrm{~Hz}, \mathrm{H} 3), 4.60(1 \mathrm{H}, \mathrm{d}, J=5.3 \mathrm{~Hz}, \mathrm{H} 2), 4.30$ (1H, dd, $J=9.8,2.5$ Hz, H6), 4.20-4.08 (3H, m, H4, H5, H6'), 2.64 (1H, d, J=5.3 Hz, OH5), 2.45 (3H, s, $\left.\mathrm{CH}_{3} \mathrm{Ar}\right), 2.28(1 \mathrm{H}, \mathrm{d}, J=2.5 \mathrm{~Hz}, \mathrm{OH} 1), 1.43,1.31$ ( 2 x $3 \mathrm{H}, 2$ x s, 2 x $\left.\left(\mathrm{CH}_{3}\right)_{2} \mathrm{CO}\right)$. IR (neat): 3406, 3312, 2999, $2945 \mathrm{~cm}^{-1}$. LRMS (ESI) $m / z: 397[\mathrm{M}+\mathrm{Na}]^{+}$.

\section{6-Azido-6-deoxy-2,3-O-isopropylidene- $\alpha, \beta$-D-mannose (5b)}

The 6-O-tosyl derivative $(2.9 \mathrm{~g}, 7.8 \mathrm{mmol})$ was placed under argon and dissolved in DMF (30 $\mathrm{mL}) . \mathrm{NaN}_{3}(2.5 \mathrm{~g}, 39 \mathrm{mmol})$ was added, and the solution was heated to $55^{\circ} \mathrm{C}$, and the reaction allowed to stir for 5 days. Reaction progress was monitored by TLC (1:1 EtOAc:Hex, v/v) and when reaction was complete, solvent was removed in vacuo, where the residue was purified by silica gel column chromatography as solid (2:1, hexane:EtOAc, v/v, Rf: 0.17$)$ to give the 6-azido derivative $\mathbf{5 b}$ (1.5 g, $6.1 \mathrm{mmol}, 80 \%)$ as a slightly yellow, viscous liquid.

${ }^{1} \mathrm{H} \mathrm{NMR}\left(\mathrm{CDCl}_{3}, 400 \mathrm{MHz}\right): \delta(\mathrm{ppm}) 5.38(1 \mathrm{H}, \mathrm{s}, \mathrm{H} 1), 4.88(1 \mathrm{H}, \mathrm{dd}, J=5.6,3.8 \mathrm{~Hz}, \mathrm{H} 3), 4.62$ $(1 \mathrm{H}, \mathrm{d}, J=5.6 \mathrm{~Hz}, \mathrm{H} 2), 4.15-4.06(2 \mathrm{H}, \mathrm{m}, \mathrm{H} 4, \mathrm{H} 5), 3.55$ (1H, dd, $J=13.0,3.5 \mathrm{~Hz}, \mathrm{H} 6), 3.44(1 \mathrm{H}$, dd, $\left.J=13.0,6.5 \mathrm{~Hz}, \mathrm{H} 6{ }^{\prime}\right), 1.47,1.33\left(2 \mathrm{x} 3 \mathrm{H}, 2 \mathrm{x} \mathrm{s}, 2 \times\left(\mathrm{CH}_{3}\right)_{2} \mathrm{CO}\right) .{ }^{13} \mathrm{C}$ NMR (DEPTQ-135\} $\left(\mathrm{CDCl}_{3}, 100 \mathrm{MHz}\right): \delta(\mathrm{ppm}) 113.0\left(\left(\mathrm{CH}_{3}\right)_{2} \mathrm{CO}\right), 101.2$ (C1), 85.4, 70.0, 79.8, 69.7 (all CH, C2, C3, $\mathrm{C} 4, \mathrm{C} 5), 54.3(\mathrm{C} 6), 26.0,24.7\left(2 \times\left(\underline{\mathrm{CH}}_{3}\right)_{2} \mathrm{CO}\right)$, Note that trace amounts of the anomer can be observed in both ${ }^{1} \mathrm{H}$ and ${ }^{13} \mathrm{C}$ NMR spectra. IR (neat): 3411, 2987, 2940, 2099 $\mathrm{cm}^{-1}$. HRMS (ESIQTOF) $m / z$ : [M+Na] $]^{+}$Calcd for $\mathrm{C}_{9} \mathrm{H}_{15} \mathrm{O}_{5} \mathrm{~N}_{3} \mathrm{Na}$ (268.0904), found 268.0901. 


\section{Ethyl 4,5:7,8-di-O-isopropylidene-3-deoxy- $\boldsymbol{\alpha}, \boldsymbol{\beta}$-D-manno-2-octulopyranosonate $(6 \mathrm{a})^{25}$}

Following the method described by Feng et al. ${ }^{25}$ the phosphonate $2(9.6 \mathrm{~g}, 29.4 \mathrm{mmol})$ was placed under argon and dissolved in dry THF (25 mL). To this was added t-BuOLi (1.9 g, $24.2 \mathrm{mmol})$ and the suspension was stirred for 15 minutes. After this time a solution of the bis-isopropylidene mannose derivative $\mathbf{1}(4.5 \mathrm{~g}, 17.3 \mathrm{mmol})$ in dry THF $(15 \mathrm{ml})$ was added via syringe to the reaction mixture. The reaction mixture was heated to $\sim 50^{\circ} \mathrm{C}$ and was then stirred for 1 hour. The reaction mixture was diluted with saturated $\mathrm{NH}_{4} \mathrm{Cl}(\sim 40 \mathrm{~mL})$ and any non-aqueous solvent was removed under reduced pressure. The aqueous residue was then extracted with EtOAc $(3 \times 60 \mathrm{~mL})$. The organic phases were combined, dried $\left(\mathrm{Na}_{2} \mathrm{SO}_{4}\right)$, filtered and concentrated under reduced pressure. The resulting residue was then used in the next step without further purification.

The residue was dissolved in THF $(30 \mathrm{~mL})$ and the reaction mixture was cooled to $0^{\circ} \mathrm{C}$ with an ice bath. To this was added 20\% $\mathrm{AcOH} / \mathrm{H}_{2} \mathrm{O}(35 \mathrm{~mL})$ and $1 \mathrm{M}$ TBAF in THF (7.1 g, $\left.27 \mathrm{~mL}, 27 \mathrm{mmol}\right)$. The reaction mixture was allowed to warm to room temperature and was stirred for 3 hours. The reaction mixture was then neutralized with the addition of $\mathrm{NaHCO}_{3}$, filtered and concentrated under reduced pressure. The residue was then dissolved in EtOAc $(100 \mathrm{~mL})$, washed with saturated $\mathrm{NaHCO}_{3}$, dried $\left(\mathrm{Na}_{2} \mathrm{SO}_{4}\right)$, filtered and concentrated under reduced pressure. The residue was purified via column chromatography (1:2, EtOAc:hexane, v/v, Rf: 0.20$)$ to give the known ${ }^{25}$ KDO compound 6a (4.4 g, $12.7 \mathrm{mmol}, 73 \%)$ as a slightly yellow, viscous liquid.

${ }^{1} \mathrm{H}$ NMR (400MHz, $\mathrm{CDCl}_{3}, \alpha / \beta$ mixture): $\delta$ (ppm) $\alpha$ anomer (major)- $4.50(1 \mathrm{H}, \mathrm{dt}, J=6.6,5.0 \mathrm{~Hz}$, $\mathrm{H} 4), 4.34$ (1H, m, H7), 4.29-4.21(3H, m, H5, $\left.\mathrm{CO}_{2} \mathrm{CH}_{2} \mathrm{CH}_{3}\right), 4.07$ (1H, dd, $\left.J=8.9,6.3 \mathrm{~Hz}, \mathrm{H} 8\right)$, $3.99(1 \mathrm{H}, \mathrm{dd}, J=8.9,4.6 \mathrm{~Hz}, \mathrm{H} 8$ '), 3.89 (1H, dd, $J=8.0,2.3 \mathrm{~Hz}, \mathrm{H} 6), 2.49$ (1H, dd, $J=14.5,6.6$ $\mathrm{Hz}, \mathrm{H} 3 \mathrm{e}), 1.88(1 \mathrm{H}, \mathrm{dd}, J=14.5,5.0 \mathrm{~Hz}, \mathrm{H} 3 \mathrm{a}), 1.45,1.41,1.36,1.35$ (4 x 3H, 4 x s, 4 x $\left.\left(\mathrm{CH}_{3}\right)_{2} \mathrm{CO}\right)$, $1.30\left(3 \mathrm{H}, \mathrm{t}, J=7.2 \mathrm{~Hz}, \mathrm{CO}_{2} \mathrm{CH}_{2} \underline{\mathrm{C}}_{3}\right) ; \beta$ anomer (minor)- $4.73(1 \mathrm{H}, \mathrm{dt}, J=8.0,2.7 \mathrm{~Hz}, \mathrm{H} 4), 4.43$ (dd, $J=8.1,2.0 \mathrm{~Hz}, \mathrm{H} 7), 4.29-4.21\left(3 \mathrm{H}, \mathrm{m}, \mathrm{H} 5, \mathrm{CO}_{2} \mathrm{CH}_{2} \mathrm{CH}_{3}\right)$, 4.09-4.04 (1H, m, H8), 4.02-3.97 (1H, m, H8'), 3.44 (1H, dd, $J=8.7,2.0 \mathrm{~Hz}, \mathrm{H} 6), 2.32$ (2H, d, $J=2.9 \mathrm{~Hz}, \mathrm{H} 3 \mathrm{a}, \mathrm{H} 3 \mathrm{e}), 1.55,1.40$, $1.38,1.35\left(4 \times 3 \mathrm{H}, 4 \times \mathrm{s}, 4 \times\left(\mathrm{CH}_{3}\right)_{2} \mathrm{CO}\right), 1.33\left(3 \mathrm{H}, \mathrm{t}, J=7.2 \mathrm{~Hz}, \mathrm{CO}_{2} \mathrm{CH}_{2} \mathrm{CH}_{3}\right) .{ }^{13} \mathrm{C} \mathrm{NMR}$ $\left\{\right.$ DEPTQ-135\} (100MHz, $\mathrm{CDCl}_{3}, \alpha / \beta$ mixture): $\delta$ (ppm) $\alpha$ isomer (major)- $169.8(\mathrm{C} 1), 109.5$, $109.3\left(2 \times\left(\mathrm{CH}_{3}\right)_{2} \mathrm{CO}\right), 94.5(\mathrm{C} 2), 74.1,71.4,70.7,70.0$ (all CH, C3, C4, C5, C7), 67.0 (C8), 62.5 $\left(\mathrm{CO}_{2} \underline{\mathrm{CH}_{2}} \mathrm{CH}_{3}\right), 32.4(\mathrm{C} 3), 27.2,27.1,25.9,25.5$ (4 x $\left.\left(\underline{\mathrm{CH}_{3}}\right)_{2} \mathrm{CO}\right), 14.1\left(\mathrm{CO}_{2} \mathrm{CH}_{2} \underline{\mathrm{CH}_{3}}\right) ; \beta$ anomer (minor)- 169.7 (C1), 109.71, $109.67\left(2 \times\left(\mathrm{CH}_{3}\right)_{2} \mathrm{CO}\right), 95.7$ (C2), 74.0, 73.5, 72.5, 70.8 (all CH, C3, $\mathrm{C} 4, \mathrm{C} 5, \mathrm{C} 7), 67.3(\mathrm{C} 8), 62.1\left(\mathrm{CO}_{2} \mathrm{CH}_{2} \mathrm{CH}_{3}\right), 31.1(\mathrm{C} 3), 27.2,26.2,25.2,24.4\left(4 \mathrm{x}\left(\mathrm{CH}_{3}\right)_{2} \mathrm{CO}\right), 14.3$ $\left(\mathrm{CO}_{2} \mathrm{CH}_{2} \mathrm{CH}_{3}\right)$. Whilst the anomeric ratio is slightly different, our NMR data is consistent with published ${ }^{1} \mathrm{H}$ and ${ }^{13} \mathrm{C}$ NMR data. ${ }^{25}$ LRMS (ESI) $m / z: 369[\mathrm{M}+\mathrm{Na}]^{+}$. 
Following the method described by Feng et al. ${ }^{25}$ the phosphonate $2(0.9 \mathrm{~g}, 2.7 \mathrm{mmol})$ was placed under argon and dissolved in dry THF (15mL). To this was added t-BuOLi (0.2 g, $2.2 \mathrm{mmol})$ and the suspension was stirred for 15 minutes. After this time a solution of the 6-azido mannose derivative $\mathbf{5 b}(0.4 \mathrm{~g}, 1.6 \mathrm{mmol})$ in dry THF $(10 \mathrm{ml})$ was added via syringe to the reaction mixture. The reaction mixture was heated to $\sim 50^{\circ} \mathrm{C}$ and was then stirred for 1 hour. The reaction mixture was diluted with saturated $\mathrm{NH}_{4} \mathrm{Cl}(\sim 20 \mathrm{~mL})$ and any non-aqueous solvent was removed under reduced pressure. The aqueous residue was then extracted with EtOAc $(3 \times 20 \mathrm{~mL})$. The organic phases were combined, dried $\left(\mathrm{Na}_{2} \mathrm{SO}_{4}\right)$, filtered and concentrated under reduced pressure. The resulting residue was then used in the next step without further purification.

The residue was dissolved in THF $(5 \mathrm{~mL})$ and the reaction mixture was cooled to $0^{\circ} \mathrm{C}$ with an ice bath. To this was added $20 \% \mathrm{AcOH} / \mathrm{H}_{2} \mathrm{O}(5.6 \mathrm{~mL})$ and $1 \mathrm{M}$ TBAF in THF $(1.6 \mathrm{~g}, 6.0 \mathrm{~mL}, 6.0$ mmol). The reaction mixture was warmed to room temperature and was stirred for 3 hours. The reaction mixture was then neutralized with the addition of $\mathrm{NaHCO}_{3}$, filtered and concentrated under reduced pressure. The residue was then dissolved in EtOAc $(50 \mathrm{~mL})$, washed with saturated $\mathrm{NaHCO}_{3}$, dried $\left(\mathrm{Na}_{2} \mathrm{SO}_{4}\right)$, filtered and concentrated under reduced pressure. The residue was purified via (1:2, EtOAc:hexane, v/v, Rf: 0.19) to give the 8-azido KDO derivative $6 \mathbf{b}$ (292 mg, $0.88 \mathrm{mmol}, 54 \%$ ) as a slightly yellow, viscous liquid.

${ }^{1} \mathrm{H}$ NMR (400MHz, $\mathrm{CDCl}_{3}, \alpha / \beta$ mixture): $\delta$ (ppm) $\alpha$ anomer (major) $-\delta 4.56-4.52(1 \mathrm{H}, \mathrm{m}, \mathrm{H} 4)$, $4.33\left(1 \mathrm{H}, \mathrm{dd}, J_{5,4}=6.2, J_{5,6}=2.4 \mathrm{~Hz}, \mathrm{H} 5\right), 4.27\left(2 \mathrm{H}, \mathrm{q}, J=6.9 \mathrm{~Hz}, \mathrm{CO}_{2} \mathrm{C}_{2} \mathrm{CH}_{3}\right), 4.10(1 \mathrm{H}$, ddd, $\left.J_{7,6}=8.2, J_{7,8}=5.9, J_{7,8}=2.9 \mathrm{~Hz}, \mathrm{H} 7\right), 4.04\left(1 \mathrm{H}, \mathrm{dd}, J_{6,7}=8.2, J_{6,5}=2.4 \mathrm{~Hz}, \mathrm{H} 6\right), 3.57(1 \mathrm{H}, \mathrm{dd}$, $\left.J_{8,8^{\prime}}=12.6, J_{8,7}=2.9 \mathrm{~Hz}, \mathrm{H} 8\right), 3.46\left(1 \mathrm{H}, \mathrm{dd}, J_{8,8}=12.6, J_{8^{\prime}, 7}=5.9 \mathrm{~Hz}, \mathrm{H} 8\right.$ '), $2.58\left(1 \mathrm{H}, \mathrm{d}, J_{3 \mathrm{e}, 4}=7.4\right.$ $\mathrm{Hz}, \mathrm{H} 3 \mathrm{e}), 1.93\left(1 \mathrm{H}, \mathrm{dd}, J_{3 \mathrm{a}, 3 \mathrm{e}}=14.2, J_{3 \mathrm{a}, 4}=5.3 \mathrm{~Hz}, \mathrm{H} 3 \mathrm{a}\right), 1.48,1.36\left(2 \times 3 \mathrm{H}, 2\right.$ x s, 2 x $\left.\left(\mathrm{CH}_{3}\right)_{2} \mathrm{CO}\right)$, $1.32\left(3 \mathrm{H}, \mathrm{t}, J=6.9 \mathrm{~Hz}, \mathrm{CO}_{2} \mathrm{CH}_{2} \mathrm{C}_{3}\right) ; \beta$ anomer (minor) $4.74\left(1 \mathrm{H}, \mathrm{ddd}, J_{4,5}=7.9, J_{4,3 \mathrm{e}}=J_{4,3 \mathrm{a}}=3.1\right.$ $\mathrm{Hz}, \mathrm{H} 4), 4.53\left(1 \mathrm{H}, \mathrm{dd}, J_{5,4}=7.9, J_{5,6}=1.9 \mathrm{~Hz}, \mathrm{H} 5\right), 4.27\left(2 \mathrm{H}, \mathrm{q}, J=6.9 \mathrm{~Hz}, \mathrm{OCH}_{2} \mathrm{CH}_{3}\right), 4.00(1 \mathrm{H}$, $\left.\operatorname{ddd}, J_{7,6}=8.8, J_{7,8}=6.0, J_{7,8}=2.9 \mathrm{~Hz}, \mathrm{H} 7\right), 3.66\left(1 \mathrm{H}, \mathrm{dd}, J_{8,8}=12.6, J_{8,7}=2.9 \mathrm{~Hz}, \mathrm{H} 8\right), 3.59-3.53$ $(1 \mathrm{H}, \mathrm{m}, \mathrm{H} 6), 3.45\left(1 \mathrm{H}, \mathrm{dd}, J_{8,8}=12.6, J_{8}, 7=6.0 \mathrm{~Hz}, \mathrm{H} 8^{\prime}\right), 2.38\left(1 \mathrm{H}, \mathrm{dd}, J_{3 \mathrm{a} / 3 \mathrm{e}}=14.2, J_{3 \mathrm{a}, 4}=3.1\right.$ $\mathrm{Hz}, \mathrm{H} 3 \mathrm{a}), 2.32\left(1 \mathrm{H}, \mathrm{d}, J_{3 \mathrm{e} / 4}=3.1 \mathrm{~Hz}, \mathrm{H} 3 \mathrm{e}\right), 1.55,1.38\left(2 \times 3 \mathrm{H}, 2\right.$ x s, 2 x $\left.\left(\mathrm{CH}_{3}\right)_{2} \mathrm{CO}\right), 1.33(3 \mathrm{H}, \mathrm{t}$, $\left.J=6.9 \mathrm{~Hz}, \mathrm{CO}_{2} \mathrm{CH}_{2} \mathrm{CH}_{3}\right) ;{ }^{13} \mathrm{C}$ NMR $\{$ DEPTQ-135 $\}$ (100MHz, $\mathrm{CDCl}_{3}, \alpha / \beta$ mixture): $\delta$ (ppm) $\alpha$ isomer (major)- $\left.169.7(\mathrm{C} 1), 109.4\left(\left(\mathrm{CH}_{3}\right)_{2} \mathrm{CO}\right)\right), 94.6(\mathrm{C} 2), 71.1,70.2,70.1,69.9$ (all CH, C4, C5, $\mathrm{C} 6, \mathrm{C} 7), 62.7\left(\mathrm{CO}_{2} \underline{\mathrm{CH}}_{2} \mathrm{CH}_{3}\right), 53.9(\mathrm{C} 8), 32.7(\mathrm{C} 3), 27.6,26.1\left(2 \times\left(\mathrm{CH}_{3}\right)_{2} \underline{\mathrm{CO}}\right), 14.1\left(\mathrm{CO}_{2} \mathrm{CH}_{2} \underline{\mathrm{CH}}_{3}\right)$; $\beta$ anomer (minor)-169.0 (C1), 109.8 (( $\left.\left(\mathrm{CH}_{3}\right)_{2} \underline{\mathrm{CO}}\right)$ ), 95.6 (C2), 72.8, 72.3, 70.9, 69.4 (all $\mathrm{CH}, \mathrm{C} 4$, C5, C6, C7), $62.3\left(\mathrm{CO}_{2} \underline{\mathrm{CH}}_{2} \mathrm{CH}_{3}\right), 54.0$ (C8), 31.2 (C3), 26.3, $24.5\left(2 \times\left(\mathrm{CH}_{3}\right)_{2} \mathrm{CO}\right), 14.2$ $\left(\mathrm{CO}_{2} \mathrm{CH}_{2} \mathrm{CH}_{3}\right)$. IR (neat) $3440,2102,1740 \mathrm{~cm}^{-1}$. HRMS (ESI-QTOF) $\mathrm{m} / z$ : $[\mathrm{M}+\mathrm{Na}]^{+}$Calcd for $\mathrm{C}_{13} \mathrm{H}_{21} \mathrm{O}_{7} \mathrm{~N}_{3} \mathrm{Na}$ (354.1272), found 354.1269. 


\section{General procedure for the reaction of commercially available aldehydes with the phosphonate 2 under standard HWE conditions, giving the results shown in Table 2.}

Following the method described by Feng et al., ${ }^{25}$ the phosphonate $2(1.0 \mathrm{~g}, 3.07 \mathrm{mmol})$ was placed under argon and dissolved in dry THF (15 mL). t-BuOLi (200 mg, $2.53 \mathrm{mmol}$ ) added and reaction mixture was then stirred for 15 minutes. The commercially available aldehyde $(1.81 \mathrm{mmol})$ was then dissolved in dry THF $(10 \mathrm{~mL})$ and was added to reaction mixture via a syringe. The reaction mixture then heated to $\sim 50^{\circ} \mathrm{C}$ and stirred for 1 hour before being cooled to room temperature, and quenched with sat. aq. $\mathrm{NH}_{4} \mathrm{Cl}(30 \mathrm{~mL})$, and then the volatiles were removed in vacuo. The residue was extracted with EtOAc $(3 \times 20 \mathrm{~mL})$, the organic phases then combined, dried $\left(\mathrm{Na}_{2} \mathrm{SO}_{4}\right)$, filtered and concentrated under vacuum.

The residue could be taken directly to the next step without further purification or it could be purified via column chromatography to give:

\section{Ethyl 2-((t-butyldimethylsilyl)oxy)-3-phenylacrylate (7a)}

Obtained by the reaction of benzaldehyde with the phosphonate 2 . The residue was purified via column chromatography (1:39, EtOAc:Hexane, v/v, Rf:0.44 \& 0.55) to give 7a (555 mg, 1.81 mmol, 96\%) as a clear liquid obtained as an 1:1 E/Z mixture (calculated by ${ }^{1} \mathrm{H}$ NMR integration). ${ }^{1} \mathrm{H} \mathrm{NMR}\left(\mathrm{CDCl}_{3}, 400 \mathrm{MHz}, \mathrm{E} / \mathrm{Z}\right.$ mixture): $\delta$ (ppm) First isomer- $7.70(2 \mathrm{H}, \mathrm{d}, J=9.62 \mathrm{~Hz}, \mathrm{H} 2$ ' $/ 6$ '), 7.37-7.22 (3H, m, H3'/5' \& H4'), 6.88 (1H, s, H3), $4.29\left(2 \mathrm{H}, \mathrm{q}, J=7.26 \mathrm{~Hz}, \mathrm{OCH}_{2} \mathrm{CH}_{3}\right), 1.38(3 \mathrm{H}$, $\left.\mathrm{t}, J=7.26 \mathrm{~Hz}, \mathrm{OCH}_{2} \mathrm{CH}_{3}\right), 0.96\left(9 \mathrm{H}, \mathrm{s}, \mathrm{SiC}\left(\mathrm{CH}_{3}\right)_{3}\right), 0.14\left(6 \mathrm{H}, \mathrm{s}, \mathrm{Si}\left(\mathrm{CH}_{3}\right)_{2}\right)$; Second isomer- 7.37$7.22\left(5 \mathrm{H}, \mathrm{m}\right.$, all ArH), $6.43(1 \mathrm{H}, \mathrm{s}, \mathrm{H} 3), 4.13\left(2 \mathrm{H}, \mathrm{q}, J=8.07 \mathrm{~Hz}, \mathrm{OC}_{2} \mathrm{CH}_{3}\right), 1.14(3 \mathrm{H}, \mathrm{t}, J=8.07$ $\left.\mathrm{Hz}, \mathrm{OCH}_{2} \mathrm{CH}_{3}\right), 1.01\left(9 \mathrm{H}, \mathrm{s}, \mathrm{SiC}\left(\mathrm{CH}_{3}\right)_{3}\right), 0.25\left(6 \mathrm{H}, \mathrm{s}, \mathrm{Si}\left(\mathrm{CH}_{3}\right)_{2}\right) .{ }^{13} \mathrm{C}$ NMR $\{\mathrm{DEPTQ}-135\}$ ( $\mathrm{CDCl}_{3}, 100 \mathrm{MHz}, \mathrm{E} / \mathrm{Z}$ mixture): $\delta$ (ppm) 165.7, 165.2 (C1), 142.3, 140.8 (C2), 134.8, 134.3 (C1'), 130.0, 128.8, 128.2 (C2'/6' or C3'/5'), 128.1 (C4'), 128.0, 127.2 (C2'/6' or C3'/5'), 120.4, 119.0 (C3), 61.4, $61.0\left(\mathrm{OC}_{2} \mathrm{CH}_{3}\right), 25.9,25.7\left(\mathrm{SiC}\left(\underline{\mathrm{CH}}_{3}\right)_{3}\right), 18.7,18.4\left(\mathrm{SiC}\left(\mathrm{CH}_{3}\right)_{3}\right), 14.5,13.9$ $\left(\mathrm{OCH}_{2} \underline{\mathrm{CH}}_{3}\right),-3.8,-4.6\left(\mathrm{Si}\left(\mathrm{CH}_{3}\right)_{2}\right)$. IR (neat): 2950, 2940, 2865, 1725, $1625 \mathrm{~cm}^{-1}$. HRMS (ESIQTOF) $m / z$ : $[\mathrm{M}+\mathrm{Na}]^{+}$Calcd for $\mathrm{C}_{17} \mathrm{H}_{26} \mathrm{O}_{3} \mathrm{SiNa}$ (329.1543), found 329.1535.

\section{Ethyl 2-((t-butyldimethylsilyl)oxy)-3-(4-methoxyphenyl)acrylate (7b)}

Obtained by the reaction of 4-methoxybenzaldehyde with the phosphonate 2. The residue was purified via column chromatography (1:19, EtOAc:Hexane, v/v, Rf:0.29 \& 0.41) to give 7b (609 $\mathrm{mg}, 1.81 \mathrm{mmol}, 100 \%)$ as a clear liquid obtained as a mixture of isomers $(2: 1$, major:minor calculated via ${ }^{1} \mathrm{H}$ NMR integration). 
${ }^{1} \mathrm{H} \mathrm{NMR}\left(\mathrm{CDCl}_{3}, 400 \mathrm{MHz}, \mathrm{E} / \mathrm{Z}\right.$ mixture): $\delta(\mathrm{ppm})$ Major isomer- $7.66(2 \mathrm{H}, \mathrm{d}, J=8.99 \mathrm{~Hz}, \mathrm{H} 2$ '/6'), $6.86\left(2 \mathrm{H}, \mathrm{d}, J=8.99 \mathrm{~Hz}, \mathrm{H} 3{ }^{\prime} / 5^{\prime}\right), 6.83(1 \mathrm{H}, \mathrm{s}, \mathrm{H} 3), 4.27\left(2 \mathrm{H}, \mathrm{q}, J=7.02 \mathrm{~Hz}, \mathrm{OC}_{2} \mathrm{CH}_{3}\right), 3.82$ $\left(3 \mathrm{H}, \mathrm{s}, \mathrm{ArOCH}_{3}\right), 1.36\left(3 \mathrm{H}, \mathrm{t}, J=7.02 \mathrm{~Hz}, \mathrm{OCH}_{2} \mathrm{CH}_{3}\right), 0.96\left(9 \mathrm{H}, \mathrm{s}, \mathrm{SiC}\left(\mathrm{CH}_{3}\right)_{3}\right), 0.13(6 \mathrm{H}, \mathrm{s}$, $\left.\mathrm{Si}\left(\mathrm{CH}_{3}\right)_{2}\right)$; Minor isomer- $7.23\left(2 \mathrm{H}, \mathrm{d}, J=8.40 \mathrm{~Hz}, \mathrm{H} 2\right.$ ' $/ 6$ '), $6.82\left(2 \mathrm{H}, \mathrm{d}, J=8.40 \mathrm{~Hz}, \mathrm{H} 3{ }^{\prime} / 5^{\prime}\right)$, $6.38(1 \mathrm{H}, \mathrm{s}, \mathrm{H} 3), 4.15\left(2 \mathrm{H}, \mathrm{q}, J=7.30 \mathrm{~Hz}, \mathrm{OC}_{2} \mathrm{CH}_{3}\right), 3.80\left(3 \mathrm{H}, \mathrm{s}, \mathrm{ArOCH}_{3}\right), 1.19(3 \mathrm{H}, \mathrm{t}, J=7.30$ $\left.\mathrm{Hz}, \mathrm{OCH}_{2} \mathrm{CH}_{3}\right), 0.99\left(9 \mathrm{H}, \mathrm{s}, \mathrm{SiC}\left(\mathrm{CH}_{3}\right)_{3}\right), 0.22\left(6 \mathrm{H}, \mathrm{s}, \mathrm{Si}\left(\mathrm{CH}_{3}\right)_{2}\right) .{ }^{13} \mathrm{C}$ NMR $\{\mathrm{DEPTQ}-135\}$ ( $\mathrm{CDCl}_{3}, 100 \mathrm{MHz}, \mathrm{E} / \mathrm{Z}$ mixture): $\delta$ (ppm) 165.9, 165.2 (C1), 159.5, 159.0 (C4'), 141.1, 139.3 (C2), 131.5, 130.3 (C2'/6'), 126.89, 126.87 (C1'), 120.9, 119.0 (C3), 113.7, 113.5 (C3'/5'), 61.3, 61.0 $\left.\left(\mathrm{O}_{\underline{C}} \mathrm{CH}_{2} \mathrm{CH}_{3}\right), 55.39,55.37\left(\mathrm{C}_{\mathrm{ar}} \underline{\mathrm{OH}}_{3}\right), 26.0,25.8\left(\mathrm{SiC}(\underline{\mathrm{CH}})_{3}\right)_{3}\right), 18.7,18.4\left(\mathrm{SiC}\left(\mathrm{CH}_{3}\right)_{3}\right), 14.5,14.1$ $\left(\mathrm{OCH}_{2} \underline{\mathrm{CH}}_{3}\right),-3.7,-4.6\left(\mathrm{Si}\left(\mathrm{CH}_{3}\right)_{2}\right)$. IR (neat): 2950, 2930, 2850, 1710, 1605, $1500 \mathrm{~cm}^{-1}$. HRMS (ESI-QTOF) $m / z$ : [M+Na] $]^{+}$Calcd for $\mathrm{C}_{18} \mathrm{H}_{28} \mathrm{O}_{4} \mathrm{SiNa}$ (359.1649), found 359.1637.

\section{Ethyl 2-((t-butyldimethylsilyl)oxy)-3-(4-chlorophenyl)acrylate (7c)}

Obtained by the reaction of 4-chlorobenzaldehyde with the phosphonate 2. The residue was purified via column chromatography (1:99, EtOAc:Hexane, v/v, Rf:0.20 \& 0.27) to give 7c (620 $\mathrm{mg}, 1.81 \mathrm{mmol}, 100 \%)$ as a clear liquid obtained as a mixture of isomers (5:2, major:minor calculated via ${ }^{1} \mathrm{H}$ NMR integration).

${ }^{1} \mathrm{H} \mathrm{NMR}\left(\mathrm{CDCl}_{3}, 400 \mathrm{MHz}, \mathrm{E} / \mathrm{Z}\right.$ mixture): $\delta(\mathrm{ppm})$ Major isomer- $7.62(2 \mathrm{H}, \mathrm{d}, J=8.46 \mathrm{~Hz}$, H2'/6'), 7.30 (2H, d, $J=8.46 \mathrm{~Hz} \mathrm{H3}$ '/5'), 6.80 (1H, s, H3), 4.28 (2H, q, J=7.09 Hz, $\left.\mathrm{OC}_{2} \mathrm{CH}_{3}\right), 1.36\left(3 \mathrm{H}, \mathrm{t}, J=7.09 \mathrm{~Hz}, \mathrm{OCH}_{2} \mathrm{C}_{3}\right), 0.94\left(9 \mathrm{H}, \mathrm{s}, \mathrm{SiC}\left(\mathrm{CH}_{3}\right)_{3}\right), 0.14(6 \mathrm{H}, \mathrm{s}$, $\left.\mathrm{Si}\left(\mathrm{CH}_{3}\right)_{2}\right)$; Minor isomer- $7.25(2 \mathrm{H}, \mathrm{d}, J=7.85 \mathrm{~Hz}, \mathrm{H} 2$ '/6'), $7.18(2 \mathrm{H}, \mathrm{d}, J=7.85 \mathrm{~Hz} \mathrm{H} 3$ '/5'), $6.34(1 \mathrm{H}, \mathrm{s}, \mathrm{H} 3), 4.13\left(2 \mathrm{H}, \mathrm{q}, J=7.57 \mathrm{~Hz}, \mathrm{OC}_{2} \mathrm{CH}_{3}\right), 1.16\left(3 \mathrm{H}, \mathrm{t}, J=7.57 \mathrm{~Hz}, \mathrm{OCH}_{2} \underline{\mathrm{H}}_{3}\right)$, $0.99\left(9 \mathrm{H}, \mathrm{s}, \mathrm{SiC}\left(\mathrm{CH}_{3}\right)_{3}\right), 0.23\left(6 \mathrm{H}, \mathrm{s}, \mathrm{Si}\left(\mathrm{CH}_{3}\right)_{2}\right) .{ }^{13} \mathrm{C} \mathrm{NMR}\{\mathrm{DEPTQ}-135\}\left(\mathrm{CDCl}_{3}, 100 \mathrm{MHz}\right.$, E/Z mixture): $\delta$ (ppm) 165.4, 164.9 (C1), 142.7, 141.2 (C2), 133.7, 132.8 (C4'), 131.1, 130.2, 128.5, 128.2 (C2'/6' \& C3'/5'), 119.3, 117.5 (C3), 61.6, $61.2\left(\mathrm{OCH}_{2} \mathrm{CH}_{3}\right), 25.9,25.7$ $\left(\mathrm{SiC}\left(\underline{\mathrm{CH}}_{3}\right)_{3}\right), 18.7,18.4\left(\mathrm{SiC}\left(\mathrm{CH}_{3}\right)_{3}\right), 14.4,14.0\left(\mathrm{OCH}_{2} \underline{\mathrm{CH}} 3\right),-3.7,-4.6\left(\mathrm{Si}\left(\mathrm{CH}_{3}\right)_{2}\right)$, Note that minor isomer was also observed in ${ }^{13} \mathrm{C}$ NMR spectrum. IR (neat): 2940, 2920, 2860, 1705 , $1620,1590 \mathrm{~cm}^{-1}$. HRMS (ESI-QTOF) $\mathrm{m} / z$ : $[\mathrm{M}+\mathrm{Na}]^{+} \mathrm{Calcd}$ for $\mathrm{C}_{17} \mathrm{H}_{25} \mathrm{O}_{3} \mathrm{ClSiNa}$ (363.1154), found 363.1148 .

\section{Ethyl 2-((t-butyldimethylsilyl)oxy)-3-(4-methylphenyl)acrylate (7d)}

Obtained by the reaction of $p$-tolualdehyde with the phosphonate 2 . The residue was purified via column chromatography (1:39, EtOAc:Hexane, v/v, Rf:0.34 \& 0.40) to give 7d (580 mg, 1.81 mmol, 100\%) as a clear liquid obtained as an equal $\mathrm{E} / \mathrm{Z}$ mixture (calculated by ${ }^{1} \mathrm{H}$ NMR integration). 
${ }^{1} \mathrm{H} \mathrm{NMR}\left(\mathrm{CDCl}_{3}, 400 \mathrm{MHz}, \mathrm{E} / \mathrm{Z}\right.$ mixture): $\delta(\mathrm{ppm})$ First isomer- $7.60(2 \mathrm{H}, \mathrm{d}, J=8.28 \mathrm{~Hz}, \mathrm{H} 2$ ' $/ 6$ '), $7.17\left(2 \mathrm{H}, \mathrm{d}, J=8.28 \mathrm{~Hz}, \mathrm{H} 3{ }^{\prime} / 5^{\prime}\right), 6.85(1 \mathrm{H}, \mathrm{s}, \mathrm{H} 3), 4.28\left(2 \mathrm{H}, \mathrm{q}, J=7.22 \mathrm{~Hz}, \mathrm{OC}_{2} \mathrm{CH}_{3}\right), 2.35$ $\left(3 \mathrm{H}, \mathrm{s}, \mathrm{ArCH}_{3}\right), 1.37\left(3 \mathrm{H}, \mathrm{t}, J=7.22 \mathrm{~Hz}, \mathrm{OCH}_{2} \mathrm{CH}_{3}\right), 0.97\left(9 \mathrm{H}, \mathrm{s}, \mathrm{SiC}\left(\mathrm{CH}_{3}\right)_{3}\right), 0.14(6 \mathrm{H}, \mathrm{s}$, $\left.\mathrm{Si}\left(\mathrm{CH}_{3}\right)_{2}\right)$; Second isomer- 7.14 (2H, d, $J=7.94 \mathrm{~Hz}, \mathrm{H} 2$ '/6'), 7.10 (2H, d, $J=7.94 \mathrm{~Hz}, \mathrm{H} 3$ '/5'), $6.39(1 \mathrm{H}, \mathrm{s}, \mathrm{H} 3), 4.14\left(2 \mathrm{H}, \mathrm{q}, J=7.09 \mathrm{~Hz}, \mathrm{OCH}_{2} \mathrm{CH}_{3}\right), 2.33\left(3 \mathrm{H}, \mathrm{s}, \mathrm{ArCH}_{3}\right), 1.17(3 \mathrm{H}, \mathrm{t}, J=7.09$ $\left.\mathrm{Hz}, \mathrm{OCH}_{2} \mathrm{CH}_{3}\right), 1.00\left(9 \mathrm{H}, \mathrm{s}, \mathrm{SiC}\left(\mathrm{CH}_{3}\right)_{3}\right), 0.23\left(6 \mathrm{H}, \mathrm{s}, \mathrm{Si}\left(\mathrm{CH}_{3}\right)_{2}\right) .{ }^{13} \mathrm{C}$ NMR $\{\mathrm{DEPTQ}-135\}$ $\left(\mathrm{CDCl}_{3}, 100 \mathrm{MHz}, \mathrm{E} / \mathrm{Z}\right.$ mixture): $\delta$ (ppm) 165.8, 165.2 (C1), 141.7, 140.1 (C2), 138.1, 137.0, 131.7, 131.5 (C1' \& C4'), 129.9, 129.0, 128.8, 128.7 (C2'/6' \& C3'/5'), 120.7, 119.2 (C3), 61.3, $61.0\left(\mathrm{OC}_{2} \mathrm{CH}_{3}\right), 26.0,25.7\left(\mathrm{SiC}\left(\underline{\mathrm{CH}}_{3}\right)_{3}\right), 21.5,21.3\left(\mathrm{C}_{\mathrm{ar}} \underline{\mathrm{CH}_{3}}\right), 18.7,18.4\left(\mathrm{SiC}\left(\mathrm{CH}_{3}\right)_{3}\right), 14.5,14.0$ $\left(\mathrm{OCH}_{2} \underline{\mathrm{CH}}_{3}\right),-3.7,-4.6\left(\mathrm{Si}\left(\mathrm{CH}_{3}\right)_{2}\right)$. IR (neat): 2970, 2925, 2850, 1715, $1625 \mathrm{~cm}^{-1}$. HRMS (ESIQTOF) $m / z$ : $[\mathrm{M}+\mathrm{Na}]^{+}$Calcd for $\mathrm{C}_{18} \mathrm{H}_{28} \mathrm{O}_{3} \mathrm{SiNa}$ (343.1700), found 343.1691.

\section{Ethyl 2-((t-butyldimethylsilyl)oxy)-3-(3-methoxyphenyl)acrylate (7e)}

Obtained by the reaction of 3-methoxybenzaldehyde with the phosphonate 2. The residue was purified via column chromatography (1:19, EtOAc:Hexane, v/v, Rf:0.33 \& 0.42) to give 7 e (559 $\mathrm{mg}, 1.66 \mathrm{mmol}, 92 \%$ ) as a clear liquid obtained as an equal E/Z mixture (calculated by ${ }^{1} \mathrm{H}$ NMR integration).

${ }^{1} \mathrm{H}$ NMR $\left(\mathrm{CDCl}_{3}, 400 \mathrm{MHz}, \mathrm{E} / \mathrm{Z}\right.$ mixture): $\delta$ (ppm) First isomer- 7.27-7.17 (2H, m, ArH), 6.84 $(1 \mathrm{H}, \mathrm{s}, \mathrm{H} 3), 6.83-6.76(2 \mathrm{H}, \mathrm{m}, \mathrm{ArH}), 4.13\left(2 \mathrm{H}, \mathrm{q}, J=7.34 \mathrm{~Hz}, \mathrm{OC}_{2} \mathrm{CH}_{3}\right), 3.79\left(3 \mathrm{H}, \mathrm{s}, \mathrm{ArOCH}_{3}\right)$, $1.14\left(3 \mathrm{H}, \mathrm{t}, J=7.34 \mathrm{OCH}_{2} \underline{\mathrm{H}}_{3}\right), 0.99\left(9 \mathrm{H}, \mathrm{s}, \mathrm{SiC}\left(\mathrm{CH}_{3}\right)_{3}\right), 0.23\left(6 \mathrm{H}, \mathrm{s}, \mathrm{Si}\left(\mathrm{CH}_{3}\right)_{2}\right)$; Second isomer7.27-7.17 (2H, m, ArH), 6.83-6.76 (2H, m, ArH), $6.37(1 \mathrm{H}, \mathrm{s}, \mathrm{H} 3), 4.28(2 \mathrm{H}, \mathrm{q}, J=7.27 \mathrm{~Hz}$ $\left.\mathrm{OC}_{2} \mathrm{CH}_{3}\right), 3.81\left(3 \mathrm{H}, \mathrm{s}, \mathrm{ArOCH}_{3}\right), 1.36\left(3 \mathrm{H}, \mathrm{t}, J=7.27 \mathrm{~Hz}, \mathrm{OCH}_{2} \mathrm{CH}_{3}\right), 0.95\left(9 \mathrm{H}, \mathrm{s}, \mathrm{SiC}\left(\mathrm{CH}_{3}\right)_{3}\right)$, $0.13\left(6 \mathrm{H}, \mathrm{s}, \mathrm{Si}\left(\mathrm{CH}_{3}\right)_{2}\right) .{ }^{13} \mathrm{C} \mathrm{NMR}\{\mathrm{DEPTQ}-135\}\left(\mathrm{CDCl}_{3}, 100 \mathrm{MHz}, \mathrm{E} / \mathrm{Z}\right.$ mixture $): \delta(\mathrm{ppm}) 165.5$, 165.1 (C1), 159.4, 159.2 (C3'), 142.5, 140.8 (C2), 136.0, $135.4\left(\mathrm{C} 1^{\prime}\right.$ '), 129.0, 128.9, 122.6, 121.2, 119.6, 118.9, 115.0, 114.1, 114.0, 112.7 (C2', C3, C4', C5', C6'), 61.3, $60.9\left(\mathrm{OC}_{2} \mathrm{CH}_{3}\right), 55.3$, $55.2\left(\mathrm{C}_{\mathrm{ar}} \underline{\mathrm{OH}}_{3}\right), 25.8,25.6\left(\mathrm{SiC}\left(\underline{\mathrm{CH}}_{3}\right)_{3}\right), 18.6,18.3\left(\mathrm{SiC}\left(\mathrm{CH}_{3}\right)_{3}\right), 14.3,13.8\left(\mathrm{OCH}_{2} \underline{\mathrm{CH}}_{3}\right),-3.8,-4.7$ $\left(\mathrm{Si}\left(\mathrm{CH}_{3}\right)_{2}\right)$. IR (neat): 2950, 2920, 2850, 1715, 1625, 1600, $1590 \mathrm{~cm}^{-1}$. HRMS (ESI-QTOF) $\mathrm{m} / z$ : $[\mathrm{M}+\mathrm{Na}]^{+}$Calcd for $\mathrm{C}_{18} \mathrm{H}_{28} \mathrm{O}_{4} \mathrm{SiNa}$ (359.1649), found 359.1643 .

\section{Ethyl 2-((t-butyldimethylsilyl)oxy)-5-methylhex-2-enoate (7f)}

Obtained by the reaction of isovaleraldehyde with the phosphonate 2 . The residue was purified via column chromatography (1:39, EtOAc:Hexane, v/v, Rf:0.17 \& 0.26) to give 7f (520 mg, 1.81 $\mathrm{mmol}, 100 \%)$ as a clear liquid obtained as a mixture of isomers (13:2, major:minor calculated via ${ }^{1} \mathrm{H}$ NMR integration). 
${ }^{1} \mathrm{H} \mathrm{NMR}\left(\mathrm{CDCl}_{3}, 400 \mathrm{MHz}, \mathrm{E} / \mathrm{Z}\right.$ mixture): $\delta(\mathrm{ppm})$ Major isomer- $5.51(1 \mathrm{H}, \mathrm{t}, J=7.98 \mathrm{~Hz}, \mathrm{H3})$, $4.20\left(2 \mathrm{H}, \mathrm{q}, J=7.14 \mathrm{~Hz}, \mathrm{OC}_{2} \mathrm{CH}_{3}\right), 2.35(2 \mathrm{H}, \mathrm{dd}, J=7.98,6.82 \mathrm{~Hz}, \mathrm{H} 4), 1.70-1.58(1 \mathrm{H}, \mathrm{m}, \mathrm{H} 5)$, $1.31\left(2 \mathrm{H}, \mathrm{t}, J=7.14 \mathrm{~Hz}, \mathrm{OCH}_{2} \mathrm{C}_{3}\right), 0.94\left(9 \mathrm{H}, \mathrm{s}, \mathrm{SiC}\left(\mathrm{CH}_{3}\right)_{3}\right), 0.91(6 \mathrm{H}, \mathrm{d}, J=6.64 \mathrm{~Hz}, \mathrm{H} 6), 0.12$ $\left(6 \mathrm{H}, \mathrm{s}, \mathrm{Si}\left(\mathrm{CH}_{3}\right)_{2}\right)$; Minor isomer- $6.04(1 \mathrm{H}, \mathrm{t}, J=7.45 \mathrm{~Hz}, \mathrm{H} 3), 4.19(2 \mathrm{H}, \mathrm{q}, J=7.23 \mathrm{~Hz}$, $\left.\mathrm{OC}_{2} \mathrm{CH}_{3}\right), 2.08(2 \mathrm{H}, \mathrm{dd}, J=7.45,7.06, \mathrm{H} 4), 1.70-1.58(1 \mathrm{H}, \mathrm{m}, \mathrm{H} 5), 1.30(3 \mathrm{H}, \mathrm{t}, J=7.23 \mathrm{~Hz}$, $\left.\mathrm{OCH}_{2} \underline{\mathrm{C}}_{3}\right), 0.96\left(9 \mathrm{H}, \mathrm{s}, \mathrm{SiC}\left(\mathrm{CH}_{3}\right)_{3}\right), 0.92(6 \mathrm{H}, \mathrm{d}, J=6.70 \mathrm{~Hz}, \mathrm{H} 6), 0.15\left(6 \mathrm{H}, \mathrm{s}, \mathrm{Si}\left(\mathrm{CH}_{3}\right)_{2}\right) .{ }^{13} \mathrm{C}$ NMR \{DEPTQ-135\} $\left(\mathrm{CDCl}_{3}, 100 \mathrm{MHz}\right): \delta$ (ppm) 165.0 (C1), 140.7 (C2), 124.9 (C3), 60.6 $\left(\underline{\mathrm{O}}_{2} \mathrm{CH}_{3}\right), 35.8(\mathrm{C} 4), 29.3(\mathrm{C} 5), 25.8\left(\mathrm{SiC}\left(\underline{\mathrm{CH}}_{3}\right)_{3}\right), 22.5$ (C6), $18.3\left(\mathrm{SiC}\left(\mathrm{CH}_{3}\right)_{3}\right), 14.3$ $\left(\mathrm{OCH}_{2} \underline{\mathrm{CH}}_{3}\right),-4.7\left(\mathrm{Si}\left(\mathrm{CH}_{3}\right)_{2}\right)$, Note that minor isomer was also observed in ${ }^{13} \mathrm{C} \mathrm{NMR}$ spectrum. IR (neat): 2980, 2910, 2860, 1720, 1625 $\mathrm{cm}^{-1}$. HRMS (ESI-QTOF) $\mathrm{m} / \mathrm{z}$ : [M+Na] ${ }^{+}$Calcd for $\mathrm{C}_{15} \mathrm{H}_{30} \mathrm{O}_{3} \mathrm{SiNa}(309.1856)$, found 309.1858.

\section{Ethyl 2-((t-butyldimethylsilyl)oxy)-4-ethyloct-2-enoate (7g)}

Obtained by the reaction of 2-ethylhexanal with the phosphonate 2 . The residue was purified via column chromatography (1:39, EtOAc:Hexane, v/v, Rf:0.19 \& 0.26) to give $7 \mathbf{g}(590 \mathrm{mg}, 1.80$ mmol, 98\%) as a clear liquid obtained as a mixture of isomers (20:1, major:minor calculated via ${ }^{1} \mathrm{H}$ NMR integration).

${ }^{1} \mathrm{H} \mathrm{NMR}\left(\mathrm{CDCl}_{3}, 400 \mathrm{MHz}\right): \delta(\mathrm{ppm})$ Major isomer- $5.18(1 \mathrm{H}, \mathrm{d}, J=11.14 \mathrm{~Hz}, \mathrm{H} 3), 4.20(2 \mathrm{H}, \mathrm{q}$, $\left.J=7.97 \mathrm{~Hz}, \mathrm{OC}_{2} \mathrm{CH}_{3}\right), 3.05-2.95(1 \mathrm{H}, \mathrm{m}, \mathrm{H} 4), 1.50-1.36(2 \mathrm{H}, \mathrm{m}, \mathrm{H} 1$ '), $1.31(3 \mathrm{H}, \mathrm{t}, J=7.97 \mathrm{~Hz}$, $\left.\mathrm{OCH}_{2} \underline{\mathrm{C}}_{3}\right), 1.30-1.19$ (5H, m, H4, H5 \& H6), 0.95 (9H, s, $\left.\mathrm{SiC}\left(\mathrm{CH}_{3}\right)_{3}\right), 0.88-0.83$ (6H, m, H2', $\mathrm{H} 8), 0.13\left(6 \mathrm{H}, \mathrm{s}, \mathrm{Si}\left(\mathrm{CH}_{3}\right)_{2}\right)$; Minor isomer- $5.77(1 \mathrm{H}, \mathrm{d}, J=10.21 \mathrm{~Hz}, \mathrm{H} 3), 4.19(2 \mathrm{H}, \mathrm{q}, J=7.01$ $\left.\mathrm{Hz}, \mathrm{OC}_{2} \mathrm{CH}_{3}\right), 2.58-2.49$ (1H, m, H4), 1.50-1.36 (2H, m, H1'), 1.33-1.19 (8H, m, $\mathrm{OCH}_{2} \mathrm{CH}_{3}, \mathrm{H} 4$, H5 \& H6), 0.95 (9H, s, $\left.\mathrm{SiC}\left(\mathrm{CH}_{3}\right)_{3}\right), 0.88-0.83$ (6H, m, H2', H8), $0.12\left(6 \mathrm{H}, \mathrm{s}, \mathrm{Si}\left(\mathrm{CH}_{3}\right)_{2}\right) .{ }^{13} \mathrm{C} \mathrm{NMR}$ \{DEPTQ-135\} $\left(\mathrm{CDCl}_{3}, 100 \mathrm{MHz}\right): \delta(\mathrm{ppm}) 165.1(\mathrm{C} 1), 140.5(\mathrm{C} 2), 131.0(\mathrm{C} 3), 60.6\left(\mathrm{OC}_{2} \mathrm{CH}_{3}\right)$, 38.1 (C4), 35.5, 29.7, 28.9, (C1', C5 \& C6), $25.8\left(\mathrm{SiC}\left(\underline{\mathrm{C}} \mathrm{H}_{3}\right)_{3}\right), 23.0(\mathrm{C} 7), 18.4\left(\mathrm{SiC}\left(\mathrm{CH}_{3}\right)_{3}\right), 14.3$, 14.2, $11.9\left(\mathrm{C}^{\prime},, \mathrm{C} 8 \& \mathrm{OCH}_{2} \mathrm{CH}_{3}\right),-4.7\left(\mathrm{Si}\left(\mathrm{CH}_{3}\right)_{2}\right)$, Note that minor isomer was also observed in ${ }^{13} \mathrm{C}$ NMR spectrum. IR (neat): 2950, 2925, 2855, 1720, $1620 \mathrm{~cm}^{-1}$. HRMS (ESI-QTOF) $\mathrm{m} / z$ : $[\mathrm{M}+\mathrm{Na}]^{+}$Calcd for $\mathrm{C}_{18} \mathrm{H}_{36} \mathrm{O}_{3} \mathrm{SiNa}$ (351.2326), found 351.2318.

\section{Ethyl 2-((t-butyldimethylsilyl)oxy)-4-methylhept-2-enoate (7h)}

Obtained by the reaction of 2-methylpentanal with the phosphonate 2. The residue was purified via column chromatography (1:39, EtOAc:Hexane, v/v, Rf:0.24 \& 0.33) to give $7 \mathbf{h}(540 \mathrm{mg}, 1.81$ $\mathrm{mmol}, 100 \%)$ as a clear liquid obtained as a mixture of isomers (16:1, major:minor calculated via ${ }^{1} \mathrm{H}$ NMR integration). 
${ }^{1} \mathrm{H} \mathrm{NMR}\left(\mathrm{CDCl}_{3}, 400 \mathrm{MHz}\right): \delta(\mathrm{ppm})$ Major isomer- $5.25(1 \mathrm{H}, \mathrm{d}, J=10.29 \mathrm{~Hz}, \mathrm{H} 3), 4.21(2 \mathrm{H}, \mathrm{q}$, $\left.J=7.08 \mathrm{~Hz}, \mathrm{OC}_{2} \mathrm{CH}_{3}\right), 3.21-3.11(1 \mathrm{H}, \mathrm{m}, \mathrm{H} 4), 1.31\left(3 \mathrm{H}, \mathrm{t}, J=7.08 \mathrm{~Hz}, \mathrm{OCH}_{2} \underline{\mathrm{H}}_{3}\right), 1.29-1.18$ (4H, m, H5 \& H6), 0.98 (3H, d, J = 6.72 Hz, H1'), 0.94 (9H, s, SiC(CH 3$\left.)_{3}\right), 0.87$ (3H, t, J=6.90 $\mathrm{Hz}, \mathrm{H} 7), 0.12\left(6 \mathrm{H}, \mathrm{s}, \mathrm{Si}\left(\mathrm{CH}_{3}\right)_{2}\right)$; Minor isomer- $5.81(1 \mathrm{H}, \mathrm{d}, J=10.09 \mathrm{~Hz}, \mathrm{H} 3), 4.21(2 \mathrm{H}, \mathrm{q}, J=$ $\left.7.25 \mathrm{~Hz}, \mathrm{OCH}_{2} \mathrm{CH}_{3}\right), 2.75-2.66(1 \mathrm{H}, \mathrm{m}, \mathrm{H} 4), 1.31\left(3 \mathrm{H}, \mathrm{t}, J=7.25 \mathrm{~Hz}, \mathrm{OCH}_{2} \underline{\mathrm{H}}_{3}\right), 1.29-1.18(5 \mathrm{H}$, m, H4, H5 \& H6), 0.97 (3H, d, $J=6.87 \mathrm{~Hz}, \mathrm{H} 1$ '), $0.94\left(9 \mathrm{H}, \mathrm{s}, \mathrm{SiC}\left(\mathrm{CH}_{3}\right)_{3}\right), 0.86(3 \mathrm{H}, \mathrm{t}, J=6.35$ $\mathrm{Hz}, \mathrm{H} 7), 0.16\left(6 \mathrm{H}, \mathrm{s}, \mathrm{Si}\left(\mathrm{CH}_{3}\right)_{2}\right) .{ }^{13} \mathrm{C}$ NMR $\{\mathrm{DEPTQ}-135\}\left(\mathrm{CDCl}_{3}, 100 \mathrm{MHz}\right): \delta(\mathrm{ppm}) 165.0(\mathrm{C} 1)$, $139.4(\mathrm{C} 2), 132.3(\mathrm{C} 3), 60.6\left(\mathrm{OCH}_{2} \mathrm{CH}_{3}\right), 40.2(\mathrm{C} 5), 31.1(\mathrm{C} 4), 25.8\left(\mathrm{SiC}\left(\underline{\mathrm{CH}}_{3}\right)_{3}\right), 21.4(\mathrm{C} 1$ '), 20.7 (C6), $18.4\left(\mathrm{SiC}\left(\mathrm{CH}_{3}\right)_{3}\right), 14.32,14.27\left(\mathrm{C} 7 \& \mathrm{OCH}_{2} \mathrm{CH}_{3}\right),-4.7,-4.8\left(\mathrm{Si}\left(\mathrm{CH}_{3}\right)_{2}\right)$, Note that minor isomer was also observed in ${ }^{13} \mathrm{C}$ NMR spectrum. IR (neat): 2970, 2920, 2860, 1710, $1630 \mathrm{~cm}^{-1}$. HRMS (ESI-QTOF) $m / z$ : [M+Na] $]^{+}$Calcd for $\mathrm{C}_{16} \mathrm{H}_{32} \mathrm{O}_{3} \mathrm{SiNa}$ (323.2013), found 323.2012.

\section{The following procedure is the typical method for the desilylation of the HWE adducts, followed by neutralisation, as depicted in Scheme 2:}

The HWE adduct $7(1.0 \mathrm{mmol})$ or crude material from the HWE reaction described above was dissolved in THF $(5.6 \mathrm{~mL})$ and cooled to $\sim 0^{\circ} \mathrm{C}$ with an ice bath. $20 \% \mathrm{AcOH} / \mathrm{H}_{2} \mathrm{O}(6.0 \mathrm{~mL})$ was added followed by 1M TBAF/THF solution (1.2 g, $4.7 \mathrm{~mL}, 4.7 \mathrm{mmol})$. The reaction mixture was allowed to warm to room temperature and stirred until starting material disappeared via TLC analysis (usually $\sim 30$ minutes). The reaction mixture was then neutralised with the addition of solid $\mathrm{NaHCO}_{3}$ (to $\mathrm{pH} \approx 8$ ), and then filtered to remove solids. The crude mixture was concentrated in vacuo, and then diluted with EtOAc $(50 \mathrm{~mL})$, washed with sat. aq. $\mathrm{NaHCO}_{3}$, dried $\left(\mathrm{Na}_{2} \mathrm{SO}_{4}\right)$, and concentrated in vacuo. The residue was then purified via column chromatography, as described below for each product.

\section{Ethyl 5-methyl-2-oxohexanoate (8a)}

Obtained 8a (139 mg, $0.81 \mathrm{mmol}, 81 \%$ ) as a clear, viscous liquid from the HWE adduct $7 \mathbf{f}$ after column chromatography (1:2, EtOAc:Hexane, v/v, Rf:0.64). ${ }^{1} \mathrm{H} \mathrm{NMR}\left(\mathrm{CDCl}_{3}, 400 \mathrm{MHz}\right): \delta(\mathrm{ppm})$ $4.29\left(2 \mathrm{H}, \mathrm{q}, J=7.08 \mathrm{~Hz}, \mathrm{OC}_{2} \mathrm{CH}_{3}\right), 2.80(2 \mathrm{H}, \mathrm{dd}, J=7.15,7.79 \mathrm{~Hz}, \mathrm{H} 3), 2.24-1.99$ (1H, m, H5), 1.53-1.46 (2H, m, H4), 1.34 (3H, t, $J=7.08$ Hz, $\left.\mathrm{OCH}_{2} \mathrm{CH}_{3}\right), 0.89,0.88$ (2 x 3H, 2 x s, H1', H6).

${ }^{13} \mathrm{C}$ NMR $\left\{\right.$ DEPTQ-135\} $\left(\mathrm{CDCl}_{3}, 100 \mathrm{MHz}\right): \delta(\mathrm{ppm}) 195.0(\mathrm{C} 2), 161.4(\mathrm{C} 1), 62.4\left(\mathrm{OCH}_{2} \mathrm{CH}_{3}\right)$, $37.4(\mathrm{C} 3), 31.8(\mathrm{C} 4), 27.7(\mathrm{C} 5), 22.3(\mathrm{C} 1$ ', $\mathrm{C} 6), 14.1\left(\mathrm{OCH}_{2} \underline{\mathrm{CH}}_{3}\right)$. Note that trace amounts of enol tautomer can be observed in both ${ }^{1} \mathrm{H}$ and ${ }^{13} \mathrm{C}$ NMR spectra. HRMS (ESI-QTOF) $m / z$ : $[\mathrm{M}+\mathrm{Na}]^{+}$ Calcd for $\mathrm{C}_{9} \mathrm{H}_{16} \mathrm{O}_{3} \mathrm{Na}$ (195.0992), found 195.0995. 


\section{Ethyl 4-ethyl-2-oxooctanoate (8b)}

Obtained $\mathbf{8 b}$ (158 mg, $0.74 \mathrm{mmol}, 74 \%)$ as a clear, viscous liquid from the HWE adduct $\mathbf{7 g}$ after column chromatography (1:2, EtOAc:Hexane, v/v, Rf:0.61). ${ }^{1} \mathrm{H} \mathrm{NMR}\left(\mathrm{CDCl}_{3}, 400 \mathrm{MHz}\right): \delta(\mathrm{ppm})$ $4.26\left(2 \mathrm{H}, \mathrm{q}, J=7.13 \mathrm{~Hz}, \mathrm{OCH}_{2} \mathrm{CH}_{3}\right), 2.69$ (2H, d, $\left.J=6.60 \mathrm{~Hz}, \mathrm{H} 3\right), 1.91-1.82$ (1H, m, H4), $1.38-$ $1.15\left(8 \mathrm{H}, \mathrm{m}, \mathrm{H} 1\right.$ ', H3, H5, H6 \& H7), $1.32\left(3 \mathrm{H}, \mathrm{t}, J=7.13, \mathrm{OCH}_{2} \mathrm{CH}_{3}\right), 0.83(3 \mathrm{H}, \mathrm{t}, J=7.02 \mathrm{~Hz}$, $\mathrm{H} 2$ '), $0.81(3 \mathrm{H}, \mathrm{t}, J=7.46 \mathrm{~Hz}, \mathrm{H} 8) .{ }^{13} \mathrm{C}$ NMR $\left\{\right.$ DEPTQ-135\} $\left(\mathrm{CDCl}_{3}, 100 \mathrm{MHz}\right): \delta(\mathrm{ppm}) 195.0$ (C2), $161.6(\mathrm{C} 1), 62.3\left(\mathrm{OC}_{2} \mathrm{CH}_{3}\right), 43.6$ (C3), 35.0 (C4), 33.2, 28.9, 26.4, 22.9 (C1', C5, C6 \& C7), $14.04,14.03,10.8\left(\mathrm{C}^{\prime}, \mathrm{C} 8 \& \mathrm{OCH}_{2} \mathrm{CH}_{3}\right)$. Note that trace amounts of enol tautomer can be observed in both ${ }^{1} \mathrm{H}$ and ${ }^{13} \mathrm{C}$ NMR spectra. IR (neat): 2960, 2920, 2850, 1720 $\mathrm{cm}^{-1}$. HRMS (ESIQTOF) $m / z$ : $[\mathrm{M}+\mathrm{Na}]^{+}$Calcd for $\mathrm{C}_{12} \mathrm{H}_{22} \mathrm{O}_{3} \mathrm{Na}$ (237.1461), found 237.1464.

\section{Ethyl 4-methyl-2-oxoheptanoate (8c)}

Obtained 8c (171 mg, $0.91 \mathrm{mmol}, 91 \%)$ as a clear, viscous liquid from the HWE adduct $\mathbf{7 h}$ after column chromatography (1:2, EtOAc:Hexane, v/v, Rf:0.61). ${ }^{1} \mathrm{H} \mathrm{NMR}\left(\mathrm{CDCl}_{3}, 400 \mathrm{MHz}\right): \delta(\mathrm{ppm})$ $4.27\left(2 \mathrm{H}, \mathrm{q}, J=7.19 \mathrm{~Hz}, \mathrm{OC}_{2} \mathrm{CH}_{3}\right), 2.77(1 \mathrm{H}, \mathrm{dd}, J=16.69,5.90 \mathrm{~Hz}, \mathrm{H} 3 \mathrm{a}), 2.58(1 \mathrm{H}, \mathrm{dd}, J=$ 16.69, 8.25 Hz, H3b), 2.02 (1H, m, H4), 1.32 (3H, t, $\left.J=7.19, \mathrm{OCH}_{2} \underline{\mathrm{H}}_{3}\right), 1.31-1.11$ (4H, m, H5 \& H6), $0.88\left(3 \mathrm{H}, \mathrm{d}, J=6.77 \mathrm{~Hz}, \mathrm{H1}{ }^{\prime}\right), 0.85(3 \mathrm{H}, \mathrm{t}, J=6.92 \mathrm{~Hz}, \mathrm{H} 7) .{ }^{13} \mathrm{C}$ NMR $\{$ DEPTQ-135\} $\left(\mathrm{CDCl}_{3}, 100 \mathrm{MHz}\right): \delta(\mathrm{ppm}) 194.7(\mathrm{C} 2), 161.5(\mathrm{C} 1), 62.4\left(\mathrm{OCH}_{2} \mathrm{CH}_{3}\right), 46.4(\mathrm{C} 3), 39.1$ (C5), 28.6 (C4), $20.0(\mathrm{C} 6), 19.8\left(\mathrm{OCH}_{2} \underline{\mathrm{CH}}_{3}\right), 14.1,14.1$ (C1'\& C7). Note that trace amounts of enol tautomer can be observed in both ${ }^{1} \mathrm{H}$ and ${ }^{13} \mathrm{C}$ NMR spectra. IR (neat): 2950, 2925, 2880, $1715 \mathrm{~cm}^{-1}$. HRMS (ESI-QTOF) $m / z$ : [M+Na $]^{+}$Calcd for $\mathrm{C}_{10} \mathrm{H}_{18} \mathrm{O}_{3} \mathrm{Na}$ (209.1148), found 209.1148.

\section{Ethyl 2-benzyl-4-hydroxy-5-0xo-3-phenyl-2,5-dihydrofuran-2-carboxylate (9a)}

Obtained 9a (139 mg, $0.41 \mathrm{mmol}, 82 \%$ ) as a white solid from the HWE adduct 7a after column chromatography (1:4, EtOAc:Hexane, v/v, Rf:0.18). ${ }^{1} \mathrm{H}$ NMR $\left(\mathrm{CDCl}_{3}, 400 \mathrm{MHz}\right): \delta(\mathrm{ppm}) 7.72$ (2H, d, J=7.45 Hz, H2'/6'), 7.51-7.39 (3H, m, H3'/5' \& H4'), 7.19-7.09 (3H, m, H2',/6' '\& H4' '), $6.85\left(2 \mathrm{H}, \mathrm{d}, J=8.11 \mathrm{~Hz}, \mathrm{H} 3\right.$ ''/5' ') $4.27\left(2 \mathrm{H}, \mathrm{q}, J=7.26 \mathrm{~Hz}, \mathrm{OC}_{2} \mathrm{CH}_{3}\right) 3.69(1 \mathrm{H}, \mathrm{d}, J=13.56$, $\mathrm{H} 7 \mathrm{a}), 3.58(1 \mathrm{H}, \mathrm{d}, J=13.56, \mathrm{H} 7 \mathrm{~b}), 1.23\left(3 \mathrm{H}, \mathrm{t}, J=7.26 \mathrm{~Hz}, \mathrm{OCH}_{2} \mathrm{CH}_{3}\right) .{ }^{13} \mathrm{C} \mathrm{NMR}$ \{DEPTQ$135\}\left(\mathrm{CDCl}_{3}, 100 \mathrm{MHz}\right) \delta(\mathrm{ppm}) 169.2,168.9$ (C5 \& C6), 138.8 (C3), 132.8 (C1'), 130.5 (C2'/6'), 129.7 (C4), 129.3 (C4'), 129.1 (C3'/C5'), 128.1 (C3''/5'), 127.8 (C4'”), 127.7 (C2',/6'), 127.4 (C4'”), 86.3 (C2), $63.1\left(\mathrm{O}^{\mathrm{CH}}{ }_{2} \mathrm{CH}_{3}\right), 39.2$ (C7), $14.0\left(\mathrm{OCH}_{2} \underline{\mathrm{CH}}_{3}\right)$. IR (neat): 3290, 3080, 3020, 2980, 1720, $1680 \mathrm{~cm}^{-1}$. HRMS (ESI-QTOF) $\mathrm{m} / \mathrm{z}$ : $[\mathrm{M}+\mathrm{Na}]^{+}$Calcd for $\mathrm{C}_{20} \mathrm{H}_{18} \mathrm{O}_{5} \mathrm{Na}(361.1046$ ), found 361.1041 . 
Ethyl 4-hydroxy-2-(4-methoxybenzyl)-5-oxo-3-(4-methoxyphenyl)-2,5-dihydrofuran-2-carboxylate

(9b)

Obtained 9b (147 $\mathrm{mg}, 0.37 \mathrm{mmol}, 74 \%$ ) as a white solid from the HWE adduct $7 \mathbf{b}$ after column chromatography (1:4, EtOAc:Hexane, v/v, Rf:0.33). ${ }^{1} \mathrm{H}$ NMR $\left(\mathrm{CDCl}_{3}, 400 \mathrm{MHz}\right): \delta(\mathrm{ppm}) 7.71$ (2H, d, $J=9.23 \mathrm{~Hz}, \mathrm{H} 2$ '/6'), 6.99 (2H, d, $J=9.23 \mathrm{~Hz}, \mathrm{H} 3$ '/5'), 6.77 (2H, d, J = 8.06 Hz, H2' '/6' '), $6.65\left(2 \mathrm{H}, \mathrm{d}, J=8.06 \mathrm{~Hz}, \mathrm{H} 3\right.$ '”/5') $, 4.25\left(2 \mathrm{H}, \mathrm{q}, J=6.90 \mathrm{~Hz}, \mathrm{OC}_{2} \mathrm{CH}_{3}\right), 3.87,3.71$ (2 x 3H, 2 x s, $\left.2 \times \mathrm{ArOCH}_{3}\right), 3.60(1 \mathrm{H}, \mathrm{d}, J=14.33 \mathrm{~Hz}, \mathrm{H} 7 \mathrm{a}), 3.51(1 \mathrm{H}, \mathrm{d}, J=14.33 \mathrm{~Hz}, \mathrm{H} 7 \mathrm{~b}), 1.22(3 \mathrm{H}, \mathrm{t}, J$ $\left.=6.90 \mathrm{~Hz}, \mathrm{OCH}_{2} \mathrm{CH}_{3}\right) .{ }^{13} \mathrm{C} \mathrm{NMR}\{\mathrm{DEPTQ}-135\}\left(\mathrm{CDCl}_{3}, 100 \mathrm{MHz}\right) \delta(\mathrm{ppm}) 169.4,169.2(\mathrm{C} 5 \&$ C6), 160.2, 158.8 (C4' \& C4' '), 137.4 (C3), 131.5, 129.5 (C3'/5' \& C3''/C5', ), 128.1 (C4), 124.8, 122.4 (C1' \& C1' '), 114.5, 113.5 (C2'/6' \& C2'”/6' '), 86.2 (C2), $63.0\left(\mathrm{OC}_{2} \mathrm{CH}_{3}\right)$, 55.5, 55.2 (2 x ArOCH 3$), 38.6(\mathrm{C} 7), 14.0\left(\mathrm{OCH}_{2} \underline{\mathrm{CH}}_{3}\right)$. IR (neat): 3290, 3005, 2950, 2920, 2830, 1760, 1730, 1700, $1605 \mathrm{~cm}^{-1}$. HRMS (ESI-QTOF) $\mathrm{m} / z$ : $[\mathrm{M}+\mathrm{Na}]^{+}$Calcd for $\mathrm{C}_{22} \mathrm{H}_{22} \mathrm{O}_{7} \mathrm{Na}$ (421.1258), found 421.1254 .

\section{Ethyl 2-(4-chlorobenzyl)-3-(4-chlorophenyl)-4-hydroxy-5-oxo-2,5-dihydrofuran-2-carboxylate (9c)}

Obtained 9c (126 mg, $0.31 \mathrm{mmol}, 62 \%)$ as a white amorphous mass from the HWE adduct 7c after column chromatography (1:4, EtOAc:Hexane, v/v, Rf: 0.19). ${ }^{1} \mathrm{H}$ NMR $\left(\mathrm{CDCl}_{3}, 400 \mathrm{MHz}\right): \delta$ (ppm) 7.67 (2H, d, $J=8.75 \mathrm{~Hz}, \mathrm{H} 2^{\prime} / 6$ ') 7.44 (2H, d, $J=8.75 \mathrm{~Hz}, \mathrm{H} 3$ '/5'), 7.11 (2H, d, $J=8.89$ Hz, H2"'/6"'), 7.01 (1H, brs, OH4), 6.77 (2H, d, J=8.89 Hz, H3',/5' '), 4.27 (2H, q, J= $7.12 \mathrm{~Hz}$, $\left.\mathrm{OC}_{2} \mathrm{CH}_{3}\right) 3.64(1 \mathrm{H}, \mathrm{d}, J=14.58 \mathrm{~Hz}, \mathrm{H} 7 \mathrm{a}), 3.51(1 \mathrm{H}, \mathrm{d}, J=14.58 \mathrm{~Hz}, \mathrm{H} 7 \mathrm{~b}), 1.23(3 \mathrm{H}, \mathrm{t}, J=7.11$ $\left.\mathrm{Hz}, \mathrm{OCH}_{2} \mathrm{CH}_{3}\right) .{ }^{13} \mathrm{C}$ NMR $\{\mathrm{DEPTQ}-135\}\left(\mathrm{CDCl}_{3}, 100 \mathrm{MHz}\right) \delta(\mathrm{ppm}) 168.68,168.67$ (C5 \& C6), 139.3, 135.5 (C4' \& C4'”), 133.6 (C3), 131.7, 129.5 (C3'/5' \& C3''/C5'), 131.2 (C4), 129.0, 128.5 (C2'/6' \& C2''/6' '), 128.0, 126.3 (C1' \& C1' '), 85.9 (C2), $63.4\left(\mathrm{OCH}_{2} \mathrm{CH}_{3}\right), 38.7$ (C7), 14.0 $\left(\mathrm{OCH}_{2} \underline{\mathrm{CH}}_{3}\right)$. HRMS (ESI-QTOF) $m / z$ : $[\mathrm{M}+\mathrm{Na}]^{+}$Calcd for $\mathrm{C}_{20} \mathrm{H}_{16} \mathrm{O}_{5} \mathrm{Cl}_{2} \mathrm{Na}$ (429.0267), found 429.0266 .

\section{Ethyl 4-hydroxy-2-(4-methylbenzyl)-5-oxo-3-(4-methylphenyl)-2,5-dihydrofuran-2-carboxylate (9d)}

Obtained 9d (70 mg, $0.19 \mathrm{mmol}, 38 \%)$ as a white solid from the HWE adduct $7 \mathbf{d}$ after column chromatography (1:4, EtOAc:Hexane, v/v, Rf:0.14). ${ }^{1} \mathrm{H}$ NMR $\left(\mathrm{CDCl}_{3}, 400 \mathrm{MHz}\right): \delta$ (ppm) 7.98 (2H, d, $J=8.06 \mathrm{~Hz}, \mathrm{H} 2$ '/6'), 7.62 (2H, d, $J=8.06 \mathrm{~Hz}, \mathrm{H} 3$ '/5'), 7.25 (2H, d, J = 8.06 Hz, H2'”/6' '), $7.09\left(2 \mathrm{H}, \mathrm{d}, J=8.06 \mathrm{~Hz}, \mathrm{H} 3\right.$ '' $/ 5$ ' '), $4.60\left(2 \mathrm{H}, \mathrm{q}, J=7.19 \mathrm{~Hz}, \mathrm{OC}_{2} \mathrm{CH}_{3}\right) 3.98(1 \mathrm{H}, \mathrm{d}, J=14.17$ $\mathrm{Hz}, \mathrm{H} 7 \mathrm{a}), 3.88(1 \mathrm{H}, \mathrm{d}, J=14.17 \mathrm{~Hz}, \mathrm{H} 7 \mathrm{~b}) 2.76,2.58\left(2 \times 3 \mathrm{H}, 2\right.$ x s, 2 x $\left.\mathrm{ArCH}_{3}\right), 1.56(3 \mathrm{H}, \mathrm{t}, J=$ $\left.7.08 \mathrm{~Hz}, \mathrm{OCH}_{2} \mathrm{CH}_{3}\right) .{ }^{13} \mathrm{C}$ NMR $\{$ DEPTQ-135 $\}\left(\mathrm{CDCl}_{3}, 100 \mathrm{MHz}\right) \delta(\mathrm{ppm}) 169.4,169.1$ (C5 \& C6), 139.6, 138.3 (C4' \& C4' '), 136.9 (C3), 130.4, 129.8 (C3'/5' \& C3''/C5', ), 129.7 (C4), 128.8, 127.8 (C2'/6' \& C2'"/6' '), 128.2, 126.9 (C1' \& C1' '), 86.3 (C2), $63.0\left(\mathrm{OCL}_{2} \mathrm{CH}_{3}\right), 39.0$ (C7), 
21.6, 21.2 (2 x $\left.\mathrm{Ar}_{\underline{C}}{ }_{3}\right), 14.0\left(\mathrm{OCH}_{2} \underline{\mathrm{CH}}_{3}\right)$. IR (neat): 3300, 3025, 3010, 2995, 2930, 1730, 1710, $1650 \mathrm{~cm}^{-1}$. HRMS (ESI-QTOF) $\mathrm{m} / z$ : $[\mathrm{M}+\mathrm{Na}]^{+}$Calcd for $\mathrm{C}_{22} \mathrm{H}_{22} \mathrm{O}_{5} \mathrm{Na}$ (389.1359), found 389.1352 .

\section{Ethyl 4-hydroxy-2-(3-methoxybenzyl)-5-oxo-3-(3-methoxyphenyl)-2,5-dihydrofuran-2-carboxylate}

(9e)

Obtained 9e (155 mg, $0.39 \mathrm{mmol}, 78 \%)$ as an off-white amorphous mass from the HWE adduct 7e after column chromatography (1:4, EtOAc:Hexane, v/v, Rf:0.14). ${ }^{1} \mathrm{H}$ NMR $\left(\mathrm{CDCl}_{3}, 400 \mathrm{MHz}\right)$ : $\delta(\mathrm{ppm}) 7.38(1 \mathrm{H}, \mathrm{t}, J=8.24 \mathrm{~Hz}, \mathrm{H} 5$ ') 7.32 (1H, brs, H2'), 7.24 (1H, m, H4' or H6'), 7.04 (1H, t, $J=8.04 \mathrm{~Hz}, \mathrm{H} 5$ ' '), 6.95 (1H, m, $1.86 \mathrm{~Hz}, \mathrm{H} 4$ ' or H6'), 6.71 (1H, ddd, $J=8.04,2.46 \mathrm{~Hz}, \mathrm{H} 4$ ', or H6'), 6.46 (1H, d, J=8.04 Hz, H4'” or H6' '), 6.41 (1H, brs, OH4), 6.36 (1H, brs, H2' '), 4.28 $\left(2 \mathrm{H}, \mathrm{q}, J=7.14 \mathrm{~Hz}, \mathrm{OC}_{2} \mathrm{CH}_{3}\right), 3.84\left(3 \mathrm{H}, \mathrm{s}, \mathrm{ArOCH}_{3}\right), 3.64(1 \mathrm{H}, \mathrm{d}, J=14.56 \mathrm{~Hz}, \mathrm{H} 1 \mathrm{a}), 3.59(3 \mathrm{H}$, $\left.\mathrm{s}, \mathrm{ArOCH}_{3}\right), 3.58(1 \mathrm{H}, \mathrm{d}, J=14.56 \mathrm{~Hz}, \mathrm{H} 1 \mathrm{~b}), 1.24\left(3 \mathrm{H}, \mathrm{t}, J=7.14 \mathrm{~Hz}, \mathrm{OCH}_{2} \mathrm{CH}_{3}\right) .{ }^{13} \mathrm{C} \mathrm{NMR}$ $\left\{\right.$ DEPTQ-135\} $\left(\mathrm{CDCl}_{3}, 100 \mathrm{MHz}\right) \delta(\mathrm{ppm}) 169.00,168.96$ (C5 \& C6), 160.0, 159.2 (C3' \& C3' '), 138.9 (C3), 134.4 (C1'), 130.9 (C4), 130.1 (C5'), 129.1 (C5'), 127.4 (C1'’), 122.9 (C4'” or C6'), 120.0 (C4' or C6'), 115.4 (C2'’), 115.3 (C4' or C6'), 113.7 (C2'’ or C6'”), 113.4 (C2'), 86.3 (C2), $63.2\left(\mathrm{OC}_{2} \mathrm{CH}_{3}\right), 55.5,55.1\left(2 \times \mathrm{ArOCH}_{3}\right), 39.4(\mathrm{C} 7), 14.1\left(\mathrm{OCH}_{2} \underline{\mathrm{CH}}_{3}\right)$. IR (neat): 3280, 3060, 3000, 2940, 2840, 1750, 1725, 1700, $1600 \mathrm{~cm}^{-1}$. HRMS (ESI-QTOF) $\mathrm{m} / z$ : $[\mathrm{M}+\mathrm{Na}]^{+}$Calcd for $\mathrm{C}_{22} \mathrm{H}_{22} \mathrm{O}_{7} \mathrm{Na}(421.1258)$, found 421.1251 .

\section{Ethyl 4-hydroxy-2-(2-methylpentyl)-5-oxo-3-(4-methoxyphenyl)-2,5-dihydrofuran-2-carboxylate (14)} A mixture of the HWE adducts $7 \mathbf{b}(200 \mathrm{mg}, 0.59 \mathrm{mmol})$ and $7 \mathbf{h}(177 \mathrm{mg}, 0.59 \mathrm{mmol})$ were dissolved in THF $(5.6 \mathrm{~mL})$ and cooled to $\sim 0^{\circ} \mathrm{C}$ with an ice bath. $20 \% \mathrm{AcOH} / \mathrm{H}_{2} \mathrm{O}(6.0 \mathrm{~mL})$ was added followed by 1M TBAF/THF solution $(1.2 \mathrm{~g}, 4.7 \mathrm{~mL}, 4.7 \mathrm{mmol})$. The reaction mixture was allowed to warm to room temperature and stirred until starting materials disappeared via TLC analysis ( $\sim 2$ hour), and then neutralised by the addition of solid $\mathrm{NaHCO}_{3}$ (to $\mathrm{pH} \approx 8$ ), and stirred for an additional 1 hour. The solid was removed by filtration and the crude mixture was concentrated in vacuo, and then diluted with EtOAc $(50 \mathrm{~mL})$, washed with sat. aq. $\mathrm{NaHCO}_{3}$, dried $\left(\mathrm{Na}_{2} \mathrm{SO}_{4}\right)$, and concentrated in vacuo. The residue was then purified via column chromatography (1:4, EtOAc:Hexane, v/v) to give 8c (11 mg, $0.06 \mathrm{mmol}, 10 \%), 9 \mathbf{b}$ (56 mg, $0.14 \mathrm{mmol}, 23 \%$ ), and the title compound $14(90 \mathrm{mg}, 0.25 \mathrm{mmol}, 42 \%)$ as a mixture of diastereomers. ${ }^{1} \mathrm{H} \mathrm{NMR}\left(\mathrm{CDCl}_{3}\right.$, $400 \mathrm{MHz}$; where the same signal for the different diastereomer appears at a different resonance frequency, the second signal is given in parentheses): $\delta(\mathrm{ppm}) 7.76(7.73)(2 \mathrm{H}, \mathrm{d}, J=9.12 \mathrm{~Hz}$, H2'/6'), 6.93 (2H, d, $\left.J=9.12 \mathrm{~Hz}, \mathrm{H}^{\prime} / 5^{\prime}\right), 4.20$ (4.19) (2H, q, $\left.J=7.05 \mathrm{~Hz}, \mathrm{OC}_{2} \mathrm{CH}_{3}\right), 3.84(3 \mathrm{H}$, s, $\left.\operatorname{ArOCH}_{3}\right), 2.49$ (1H, dd, $J=14.85,4.35 \mathrm{~Hz}, \mathrm{H1a}$ "), 2.04 (1H, dd, $J=14.85,6.88 \mathrm{~Hz}, \mathrm{H} 1 \mathrm{~b}$ "), 
(2.28 (AB system, 2H, H1")), 1.50-1.31 (1H, m, H2”), 1.28-1.05 (4H, m, H3"/H4”), 1.19 (1.19) $\left(3 \mathrm{H}, \mathrm{t}, J=7.05 \mathrm{~Hz}, \mathrm{OCH}_{2} \mathrm{C}_{3}\right), 0.88(0.71)(3 \mathrm{H}, \mathrm{d}, J=6.65 \mathrm{~Hz}, \mathrm{C} 2$ '”-Me), 0.84 (0.67) (3H, t, $J=$ $6.92 \mathrm{~Hz}, \mathrm{H} 5 ”) .{ }^{13} \mathrm{C}$ NMR $\{\mathrm{DEPTQ}-135\}\left(\mathrm{CDCl}_{3}, 100 \mathrm{MHz}\right.$, where the same signal for the different diastereomer appears at a different resonance frequency, the second signal is given in parentheses): $\delta$ (ppm) 170.1, 169.5 (170.1, 169.4) (C5 \& C6), 160.2 (160.1) (C4'), 137.1 (137.0) (C3), 129.6 (129.6) (C3'/5'), 129.5 (C4), 122.4 (122.2) (C1'), 114.4 (114.3) (C2'/6'), 87.6 (87.3) (C2), 62.9 (62.8) $\left(\mathrm{OCH}_{2} \mathrm{CH}_{3}\right), 55.4$ (55.4) $\left(\mathrm{ArO}^{\mathrm{CH}}{ }_{3}\right), 40.6$ (39.9) (C1'’), 40.4 (40.4) (C3'” or C4'’), 28.1 (28.0) (C2'”), 21.3 (21.2) (C2’'-Me), 19.8 (19.7) (C3'” or C4’'), 14.3 (14.1) $\left(\mathrm{OCH}_{2} \underline{\mathrm{CH}}_{3}\right)$, 14.0(C5”). IR (neat): 3295, 2958, 2931, 2872, 1742, $1730 \mathrm{~cm}^{-1}$. HRMS (ESI-QTOF) $\mathrm{m} / z$ : $[\mathrm{M}+\mathrm{Na}]^{+}$Calcd for $\mathrm{C}_{20} \mathrm{H}_{26} \mathrm{O}_{6} \mathrm{Na}(385.1622)$, found 385.1620.

\section{Ethyl 4-hydroxy-2-(4-methoxybenzyl)-5-oxo-3-phenyl-2,5-dihydrofuran-2-carboxylate (15) and}

\section{Ethyl 4-hydroxy-2-benzyl-5-oxo-3-(4-methoxyphenyl)-2,5-dihydrofuran-2-carboxylate (16)}

A mixture of the HWE adducts $7 \mathbf{a}(330 \mathrm{mg}, 1.08 \mathrm{mmol})$ and $7 \mathbf{b}(360 \mathrm{mg}, 1.08 \mathrm{mmol})$ were dissolved in THF $(5.6 \mathrm{~mL})$ and cooled to $\sim 0^{\circ} \mathrm{C}$ with an ice bath. $20 \% \mathrm{AcOH} / \mathrm{H}_{2} \mathrm{O}(6.0 \mathrm{~mL})$ was added followed by 1M TBAF/THF solution $(1.2 \mathrm{~g}, 4.7 \mathrm{~mL}, 4.7 \mathrm{mmol})$. The reaction mixture was allowed to warm to room temperature and stirred until starting materials disappeared via TLC analysis ( $~ 4$ hour), and then neutralised by the addition of solid $\mathrm{NaHCO}_{3}$ (to $\mathrm{pH} \approx 8$ ), and stirred for an additional 1 hour. The solid was removed by filtration and the crude mixture was concentrated in vacuo, and then diluted with EtOAc $(50 \mathrm{~mL})$, washed with sat. aq. $\mathrm{NaHCO}_{3}$, dried $\left(\mathrm{Na}_{2} \mathrm{SO}_{4}\right)$, and concentrated in vacuo. The residue was then purified via column chromatography (1:9, EtOAc:Hexane, v/v) to give 9a (75 mg, $0.22 \mathrm{mmol}, 20 \%), 9 b$ (80 mg, $0.20 \mathrm{mmol}, 19 \%$ ), and a mixture of the title compounds 15 and 16 (170 mg, $0.46 \mathrm{mmol}, 43 \%)$ as an inseparable mixture of isomers in approximately equal amounts (as calculated via ${ }^{1} \mathrm{H}$ NMR integration).

${ }^{1} \mathrm{H} \mathrm{NMR}\left(\mathrm{CDCl}_{3}, 400 \mathrm{MHz}\right.$, both isomers): $\delta$ (ppm) 7.73-7.68 (4H, m, 2 x H2'/6'), 7.49-7.35 (3H, m, H3'/5' \& H4'), 7.16-7.09 (3H, m, H2',/6' '\& H4' '), 6.99 (2H, d, J=8.14 Hz, H3'/5'), 6.85 (2H, m, H3',/5'), 6.75 (2H, d, $J=8.76 \mathrm{~Hz}, \mathrm{H} 2$ ',/6' '), 6.66 (2H, d, $J=8.76 \mathrm{~Hz}, \mathrm{H} 3$ ' '/5' '), 6.55, 6.38 (2 x 1H, 2 x brs, 2 x OH4), 4.30-4.22 (4H, m, 2 x OC$\left.{ }_{2} \mathrm{CH}_{3}\right), 3.88,3.72\left(2 \times 3 \mathrm{H}, 2\right.$ x s, 2 x $\left.\mathrm{ArOCH}_{3}\right)$, 3.66, $3.62(2 \times 1 \mathrm{H}, 2 \times \mathrm{d}, J=\sim 14.57 \mathrm{~Hz}, 2 \times \mathrm{H} 7 \mathrm{a}), 3.56,3.52(2 \times 1 \mathrm{H}, 2 \times \mathrm{d}, J=\sim 14.57 \mathrm{~Hz}, 2 \times$ $\mathrm{H} 7 \mathrm{~b}), 1.22\left(6 \mathrm{H}, \mathrm{t}, J=7.38 \mathrm{~Hz}, 2 \times \mathrm{OCH}_{2} \mathrm{CH}_{3}\right) .{ }^{13} \mathrm{C} \mathrm{NMR}\{\mathrm{DEPTQ}-135\}\left(\mathrm{CDCl}_{3}, 100 \mathrm{MHz}\right.$, both isomers): $\delta$ (ppm) 169.2, 169.2, 169.1, 169.0, (2 x C5 \& C6), 160.3, 158.8 (C4' \& C4'”), 138.8, 137.3 (2 x C3), 132.9 (C1'), 131.6, 130.5 (C3'/5' \& C3''/C5'’), 129.7 (C4), 129.5 (C2'/C6'), 129.4 (C4'), 129.1, 128.1 (C3'/C5' \& C3'”/5' '), 128.0 (C4), 127.8 (C2'"/6' '), 127.4 (C4' '), 124.8, 124.3 (C1'\& C1'’), 114.6, 113.6 (C2'/6' \& C2'”/6' '), 86.4, 86.1 (2 x C2), 63.1 (2 x $\left.\mathrm{OCH}_{2} \mathrm{CH}_{3}\right), 55.5$, $55.2\left(2 \times \mathrm{ArOC}_{3}\right), 39.5,38.5(2 \times \mathrm{C} 7), 14.0\left(2 \times \mathrm{OCH}_{2} \underline{\mathrm{CH}}_{3}\right)$. 
HRMS (ESI-QTOF) $m / z$ : $[\mathrm{M}+\mathrm{H}]^{+}$Calcd for $\mathrm{C}_{21} \mathrm{H}_{21} \mathrm{O}_{6}$ (369.1333), found 369.1327.

\section{ASSOCIATED CONTENT}

\section{Supporting Information}

${ }^{1} \mathrm{H} \&{ }^{13} \mathrm{C}$ NMR spectra are included.

The Supporting Information is available free of charge on the ACS Publications website.

\section{AUTHOR INFORMATION}

\section{Corresponding Author}

*m.kiefel@griffith.edu.au

\section{ORCID}

Milton J Kiefel: 0000-0003-1030-1440

\section{Notes}

The authors declare no competing financial interest.

\section{ACKNOWLEDGMENT}

This work was supported by a Griffith University postgraduate scholarship (GUPRA) for JE, and the Institute for Glycomics.

\section{REFERENCES}

(1) Kobayashi, K.; Tanaka III, K.; Kogen, H. Recent Topics of the Natural Product Synthesis by HornerWadsworth-Emmons Reaction. Tetrahedron Lett. 2018, 59, 568.

(2) Burtoloso, A.C.B.; Dias, R.M.P.; Bernardim, B. $\alpha, \beta$-Unsaturated Diazoketones as Useful Platforms in the Synthesis of Nitrogen Heterocycles. Acc. Chem. Res. 2015, 48, 921.

(3) Al Jasem, Y.; El-Esawi, R.; Thiemann, T. Wittig- and Horner-Wadsworth-Emmons-olefination Reactions with Stabilized and Semi-Stabilized Phosphoranes and Phosphonates under non-classical Conditions. J. Chem. Res. 2014, 38, 453.

(4) Bisceglia, J.A.; Orelli, L.R. Recent Applications of the Horner-Wadsworth-Emmons Reaction to the Synthesis of Natural Products. Curr. Org. Chem. 2012, 16, 2206.

(5) Xie, C.; Luo, J.; Zhang, Y.; Zhu, L.; Hong, R. A Chiral Pentenolide-Based Unified Strategy toward Dihydrocorynantheal, Dihydrocorynantheol, Protoemetine, Protometinol, and Yohimbane. Org. Lett. 2017, 19, 3592.

(6) Poock, C.; Kalesse, M. Total Synthesis of Nannocystin Ax. Org. Lett. 2017, 19, 4536.

(7) see for example: (a) Paul, D.; Saha, S.; Goswami, R. K. Total Synthesis of Pestalotioprolide E and Structural Revision of Pestalotioprolide F. Org. Lett. 2018, 20, 4606. (b) Lu, L.; Zhang, W.; Nam, S.; Horne, D.A.; Jove, R.; Carter, R.G. Amphidinolide B: Total Synthesis, Structural Investigation, and Biological Evaluation. J. Org. Chem. 2013, 78, 2213. (c) Dias, L.C.; de Lucca, Jr., E.C. Total Synthesis of (-)-Marinisporolide C. J. Org. Chem. 2017, 82, 3019.

(8) Hong, W.P.; Noshi, M.N.; El-Awa, A.; Fuchs, P.L. Synthesis of the C1-C20 and C15-C27 Segments of Aplyronine A. Org. Lett. 2011, 13, 6342. 
(9) Rocha, D.A.; Silva, E.B.; Fortes, I.S.; Lopes, M.S.; Ferreira, R.S.; Andrade, S.F. Synthesis and Structure-Activity Relationship Studies of Cruzain and Rhodesain Inhibitors. Eur. J. Med. Chem. 2018, $157,1426$.

(10) Han, J.; Soloshonok, V.A.; Klika, K.D.; Drabowicz, J.; Wzorek, A. Chiral Sulfoxides: Advances in Asymmetric Synthesis and Problems with the Accurate Determination of the Stereochemical Outcome. Chem. Soc. Rev. 2018, 47, 1307.

(11) Zunk, M.; Williams, J.; Carter, J. Kiefel, M.J. A New Approach Towards the Synthesis of Pseudaminic Acid Analogues. Org. Biomol. Chem. 2014, 12, 2918.

(12) Williams, J.T.; Corcilius, L.; Kiefel, M.J.; Payne, R.J. Total Synthesis of Native 5,7Diacetylpseudaminic Acid from N-Acetylneuraminic Acid. J. Org. Chem. 2016, 81, 2607.

(13) Carter, J.R.; Kiefel. M.J. A New Approach to the Synthesis of Legionaminic Acid Analogues. RSC $A d v$. 2018, 8,35768 .

(14) see for example: (a) Logan, S.M.; Kelly, J.F.; Thibault, P.; Ewing, C.P.; Guerry, P. Structural Heterogeneity of Carbohydrate Modification Affects Serospecificity of Campylobacter flagellins. Mol. Microbiol. 2002, 46, 587. (b) McNally, D.J.; Aubry, A.J.; Hui, J.P.M.; Khieu, N.H.; Whitfield, D.; Ewing, C.P.; Guerry, P.; Brisson, J.R.; Logan, S.M.; Soo, E.C. Targeted Metabolomics Analysis of Campylobacter coli VC167 Reveals Legionaminic Acid Derivatives as Novel Flagellar Glycans. J. Biol. Chem. 2007, 282, 14463.

(15) see for example: (a) Knirel, Y.A.; Kocharova, N.A.; Shashkov, A.S.; Kochetkov, N.K.; Kholodkova, E.V.; Stanislavsky, E.S. Somatic Antigens of Pseudomonas Aeruginosa. Eur. J. Biochem. 1987, 166, 189. (b) Knirel, Y.A.; Helbig, J.H.; Zähringer, U. Structure of a Decasaccharide Isolated by Mild Acid Degradation and Dephosphorylation of the Lipopolysaccharide of Pseudomonas fluorescens strain ATCC 49271. Carbohydr. Res. 1996, 283, 129.

(16) see for example: (a) Kenyon, J.J.; Notaro, A.; Hsu, L.Y.; De Castro, C.; Hall, R.M. 5,7-Di-N-acetyl8-epiacinetaminic acid: A New Non-2-Ulosonic Acid Found in the K73 Capsule Produced by an Acinetobacter Baumannii Isolate from Singapore. Sci. Rep. 2017, 7, 1. (b) Tsvetkov, Y.E.; Shashkov, A.S.; Knirel, Y.A.; Zähringer, U. Synthesis and Identification in Bacterial Lipopolysaccharides of 5,7-diacetamido-3,5,7,9-tetradeoxy-D-glycero-D-galacto and D-glycero-D-talo-non-2-ulosonic acids. Carbohydr. Res. 2001, 331, 233.

(17) Schirm, M.; Soo, E.C.; Aubry, A.J.; Austin, J.; Thibault, P.; Logan, S.M. Structural, Genetic and Functional Characterization of the Flagellin Glycosylation Process in Helicobacter pylori. Mol. Microbiol. 2003, 48, 1579.

(18) Knirel, Y.A.; Rietschel, E.T.; Marre, R.; Zähringer, U. The Structure of the O-specific Chain of Legionella Pneumophila serogroup 1 Lipopolysaccharide. Eur. J. Biochem. 1994, 221, 239.

(19) Cazalet, C.; Jarraud, S.; Ghavi-Helm, Y.; Kunst, F.; Glaser, P.; Etienne, J.; Buchrieser, C. Multigenome Analysis Identifies a Worldwide Distributed Epidemic Legionella Pneumophila Clone that Emerged within a Highly Diverse Species. Genone Res. 2008, 18, 431.

(20) Knirel, Y.A.; Shevelev, S.D.; Perepelov, A.V.; Higher Aldulosonic Acids: Components of Bacterial Glycans. Mendeleev Commun. 2011, 21, 173.

(21) Zunk, M.; Kiefel, M.J. The Occurrence and Biological Significance of the $\alpha$-keto-sugars Pseudaminic Acid and Legionaminic Acid within Pathogenic Bacteria. RSC Adv. 2014, 4, 3413.

(22) Matthies, S.; Stallforth, P.; Seeberger, P.H. Total Synthesi of Legionaminic Acid as a Basis for Serological Studies. J. Am. Chem. Soc. 2015, 137, 2848.

(23) for a recent example see: Dhakal, B.; Crich, D. Synthesis and Stereocontrolled Equatorially Selective Glycosylation Reaction of a Pseudaminic Acid Donor: Importance of the Side-Chain Conformation and Regioselective Reduction of the Azide Protecting Groups. J. Am. Chem. Soc. 2018, 140, 15008, and references therein.

(24) for a recent example see: Ginter, M.; Yoneda, Y.; Schmölzer, C.; Denner, C.; Kählig, H.; Schmid, W. A Versatile De Novo Synthesis of Legionaminic Acid and 4-epi-Legionaminic acid Starting from D-Serine. Carbohydr. Res. 2019, 474, 34. 
(25) Feng, Y.; Dong, J.; Xu, F.; Liu, A.; Wang, L.; Zhang, Q.; Chai, Y. Efficient Large Scale Synthesis of 3-Deoxy-D-manno-2-octulosonic acid (KDO) and Its Derivatives. Org. Lett. 2015, 17, 2388.

(26) Winzar, R.; Phillips, J.; Kiefel, M.J. A Simple Synthesis of C-8 modified 2-Keto-3-deoxy-D-mannooctulosonic acid (KDO) Derivatives. Synlett 2010, 583.

(27) Nitta, K.; Fujita, N.; Yoshimura, T.; Arai, K.; Yamamoto, Y. Metabolic Products of Aspergillus terreus. IX. Biosynthesis of Butyrolactone Derivatives Isolated from Strains IFO 8835 and 4100. Chem. Pharm. Bull. 1983, 31, 1528.

(28) Qi, C.; Gao, W.; Wang, J.; Liu, M.; Zhang, J.; Chen, C.; Hu, Z.; Xue, Y.; Li, D.; Zhang, Q.; Lai, Y.; Zhou, Q.; Zhu, H.; Zhang, Y. Terrusnolides A-D, New Butenolides with Anti-Inflammatory Activities from an Endophytic Aspergillus from Tripterygium wilfordii. Fitoterapia 2018, 130, 134.

(29) Kiriyama, N.; Nitta, K.; Sakaguchi, Y.; Taguchi, Y.; Yamamoto, Y. Studies on the Metabolic Products of Aspergillus terreus. III. Metabolites of the Strain IFO 8835. Chem. Pharm. Bull. 1977, 25, 2593.

(30) Suzuki, M.; Hosaka, Y.; Matsushima, H.; Goto, T.; Kitamura, T.; Kawabe, K. Butyrolactone I induces cyclin B1 and causes G2/M arrest and Skipping of Mitosis in Human Prostate Cell Lines. Cancer Lett. 1999, 138, 121.

(31) Braña, M.F.; Garcia, M.L.; Lopez, B.; de Pascual-Teresa, B.; Ramos, A.; Pozuelo, J.M.; Dominguez, M.T. Synthesis and Biological Evaluation of Analogues of Butyrolactone I and Molecular Model of its Interaction with CDK2. Org. Biomol. Chem. 2004, 2, 1864.

(32) Qi, C.; Gao, W.; Guan, D.; Wang, J.; Liu, M.; Chen, C.; Zhu, H.; Zhou, Y.; Lai, Y.; Hu, Z.; Zhou, Q.; Zhang, Y. Butenolides from a Marine-derived Fungus Aspergillus terreus with Antitumour Activities Against Pancreatic Ductal Adenocarcinoma Cells. Bioorg. Med. Chem. 2018, 26, 5903.

(33) Bernier, D.; Moser, F.; Brückner, R. Synthesis and Cyclization of 3-Aryl-2-(arylacetoxy)acrylates: A Three-Step Access to Pulvinines. Synthesis 2007, 2240.

(34) recent examples include: (a) El Arba, M.; Dibrell, S.E.; Meece, F.; Frantz, D.E. Ru(II)-Catalysed Synthesis of Substituted Furans and Their Conversion to Butenolides. Org. Lett. 2018, 20, 5886; (b) Qi, C.; Peng, Y.; Wang, L.; Ren, Y.; Jiang, H. Copper-Catalysed [2+3] Cyclization of $\alpha$-Hydroxyl Ketones and Arylacetonitriles: Access to Multisubstiuted Butenolides and Oxazoles. J. Org. Chem. 2018, 83, 11926; (c) Hermann, D.; Brückner, R. Silver-Catalysed tert-Butyl 3-Oxopent-4-ynoate $\pi$ Cyclizations: Controlling the Ring Size-Hydroxypyrone or Pulvinine Formation-by Counterion and Additive Optimization. Org. Lett. 2018, 20, 7455.

(35) Boussonniere, A.; Beneteau, R.; Rouaud, J.-C.; Despiau, C.; Lebreton, J.; Denes, F. Chemoselective Access to Substituted Butenolides via a Radical Cyclization Pathway: Mechanistic Study, Limits, and Application. Pure Appl. Chem. 2016, 88, 215.

(36) Kobayashi, K.; Kuwahara, K.; Tanaka III, K.; Kunimura, R.; Kogen, H. Total Synthesis of Lissoclinolide by acid-induced lactonization of an $(E)-\alpha$-Bromo- $\gamma, \delta$-epoxy acrylate derivative. Heterocycles, 2018, 97, 1019.

(37) Fichter, F. On Synthetic p-Dialkylated Dioxyquinones. Liebigs Ann. Chem. 1908, 361, 363.

(38) Mao, B.; Fananas-Mastral, M.; Feringa, B.L. Catalytic Asymmetric Synthesis of Butenolides and Butyrolactones. Chem. Rev. 2017, 117, 10502.

(39) Aubert, S.; Katsina, T.; Arseniyadis, S. A Sequential Pd-AAA/Cross-Metathesis/Cope Rearrangement Strategy for the Stereoselective Synthesis of Chiral Butenolides. Org. Lett. 2019, 21, 2231 .

(40) Justus, K.; Herrmann, R.; Klamann, J.-D.; Gruber, G.; Hellwig, V.; Ingerl, A.; Polborn, K.; Steffan, B.; Steglich, W. Retipolides - Unusual Spiromacrolactones from the Mushrooms Retiboletus Retipes and R. ornatipes. Eur. J. Org. Chem. 2007, 5560.

(41) Armarego, W.L.F.; Chai, C.L.L. Purification of Laboratory Chemicals, $7^{\text {th }}$ edn, ButterworthHeinemann, Oxford, 2013. 
(42) Wadouachi, A.; Beaupere, D.; Uzan, R.; Stasik, I.; Ewing, D.F.; Mackenzie, G. Stereochemical control in the formation of thiazolidines from O-protected reducing sugars. Carbohydr. Res. 1994, $262,147$. 